\title{
An invariant of rational homology 3-spheres via vector fields
}

\author{
TATSURO SHIMIZU
}

\begin{abstract}
We give an alternative construction of the Kontsevich-Kuperberg-Thurston invariant for rational homology 3-spheres. This construction is a generalization of the original construction of the Kontsevich-Kuperberg-Thurston invariant. As an application, we give a Morse homotopy theoretic description of the Kontsevich-Kuperberg-Thurston invariant (close to a description by Watanabe).
\end{abstract}

$57 \mathrm{M} 27$

\section{Introduction}

M Kontsevich [6], S Axelrod and I M Singer [2] proposed the Chern-Simons perturbation theory and gave a topological invariant of 3-manifolds with a flat connection. Based on Kontsevich's work, G Kuperberg and D Thurston [7] constructed a topological invariant of rational homology 3-spheres. They showed that this invariant is a universal finite type invariant for integral homology 3 -spheres by showing surgery formulas. C Lescop obtained surgery formulas of other types in [9] and [10]. Lescop revisited the Kontsevich-Kuperberg-Thurston construction more precisely and gave a more direct proof of well-definedness of this invariant in [8].

K Fukaya [3] constructed a topological invariant of 3-manifolds with two local coefficients using Morse functions. Fukaya's invariant is closely related to the 2-loop term (ie the graph $\theta$ term) of the Chern-Simons perturbation theory. M Futaki [4] pointed out that Fukaya's invariant depends on the choice of Morse functions.

$\mathrm{T}$ Watanabe [14] gave an invariant of integral homology 3-spheres without local coefficients using Morse functions. He also investigated higher loop graphs (and broken graphs), and then he defined a topological invariant of homology 3-spheres corresponding to higher order terms of the Chern-Simons perturbation theory. In this paper, we extend his construction to any rational homology 3-sphere. The construction of the 2-loop term of Watanabe's invariant is related to the construction of a Morse propagator constructed by Lescop [11].

Fukaya's construction is inspired by the construction of the 2-loop term of the ChernSimons perturbation theory, and he conjectured in [3, Section 8$]$ that his invariant is 
related to the 2-loop term of the Chern-Simons perturbation theory. Watanabe also conjectured in [14, Conjecture 2.14] that his invariant is related to Axelrod and Singer's invariant [2] or Kontsevich's invariant [6].

Let $Y$ be a rational homology 3 -sphere with a basepoint $\infty \in Y$. The KontsevichKuperberg-Thurston invariant and Watanabe's Morse homotopy invariant are defined by using extra pieces of information from $Y$ : a framing of $T(Y \backslash \infty)$ and a collection of Morse functions on $Y$, respectively.

In this paper, we give an alternative construction of the Kontsevich-Kuperberg-Thurston invariant by using a collection of appropriate 2-forms on the unit sphere bundle of the tangent bundle of $Y \backslash \infty$. In particular, we give a construction of such a collection of 2 -forms by using a collection of vector fields on $Y \backslash \infty$. We prove that it is possible to regard the constructions of the Kontsevich-Kuperberg-Thurston invariant and Watanabe's Morse homotopy invariant as special cases of our construction. In fact, a framing gives us a nonvanishing vector field and a Morse function gives us a gradient vector field.

We remark that our construction of the principal term of the degree- 1 part is related to Lescop's invariant [12] for rational homology 3-spheres with nonvanishing vector fields.

The organization of this paper is as follows. In Section 2, we prepare some notation. In Section 3, we review the Kontsevich-Kuperberg-Thurston invariant. In Section 4, we give an alternative construction of this invariant, and use it to give a Morse homotopy theoretic description of the invariant. In Section 5, we first review some lemmas from [8] used in Section 3 and Section 4, and then we prove the main theorems. In the Appendix, we give a more direct proof of Proposition 5.3 in the case $n=1$.

Acknowledgments The author would like to thank Professor Mikio Furuta for his encouragement and for helpful comments and suggestions in particular about Morse functions on punctured manifolds. The author would also like to thank Professor Tadayuki Watanabe for his helpful comments and suggestions for an earlier draft and his patient explanation of the detail of the construction of his invariant. The author also expresses his appreciation to Professor Christine Lescop for her kind and helpful comments and suggestions to improve an earlier draft. The author is deeply indebted to the referee for his/her valuable comments and suggestions. Due to his/her suggestion, the organization of this paper, some conventions and the proof of Lemma 5.11(4) were refined. The description of the proof of Lemma 4.5 is due to Lescop and the referee.

\section{Notation and some remarks}

In this article, all manifolds are smooth and oriented. Homology and cohomology are with rational coefficients. Let $c$ be a $\mathbb{Q}$-linear sum of finitely many maps from compact 
$k$-dimensional manifolds with corners to a topological space $X$. We consider $c$ as a $k$-chain of $X$ via appropriate (not unique) triangulations of each $k$-manifold.

Let $Y$ be a submanifold of a manifold $X$. Let $c=\sum_{i} a_{i}\left(f_{i}: \Sigma_{i} \rightarrow X\right)$ be a chain of $X$, where $f_{i}: \Sigma_{i} \rightarrow X$ are smooth maps from compact manifolds with corners and $a_{i}$ are rational numbers. If $f_{i}$ is transverse to $Y$ for each $i$, then we say that $c$ is transverse to $Y$.

Let us denote by $\underline{\mathbb{R}}^{k}$ the trivial vector bundle over an appropriate base space with rank $k \in \mathbb{N}$. For a real vector space $X$, we define $S(X)$ by

$$
S(X)=(X \backslash\{0\}) / \mathbb{R}_{+} .
$$

We denote $S(X)$ by $S X$. For a real vector bundle $E \rightarrow B$ over a manifold $B$, we denote by $S E$ or $S(E)$ the fiber bundle where the fiber over $x \in B$ is $S E_{x}$. When $E$ has an Euclidean metric, $S E$ is the unit sphere bundle of $E$.

When $B$ is a submanifold of a manifold $A$, we denote by $B \ell(A, B)$ the manifold given by a real blowing up of $A$ along $B$. Namely $B \ell(A, B)=(A \backslash B) \cup S v_{B}$, where $v_{B}$ is the normal bundle of $B \subset A$ (see [7] for more details about real blowing up). Note that if a submanifold $C \subset A$ is transverse to $B$, then $B \ell(C, C \cap B)$ is a properly embedded submanifold of $B \ell(A, B)$.

Let us denote by $\Delta \subset A^{n}$ the fat diagonal of the $n^{\text {th }}$ power of a manifold $A$ : namely, $\Delta=\left\{\left(x_{1}, \ldots, x_{n}\right) \in A^{n} \mid \#\left\{x_{1}, \ldots, x_{n}\right\}<n\right\}$.

\section{Conventions on orientations}

Boundaries are oriented by the outward normal first convention. Products are oriented by the order of the factors. Let $y \in B$ be a regular point of a smooth map $f: A \rightarrow B$ between smooth manifolds $A$ and $B$. Let us orient $f^{-1}(y)$ by the following rules: $T_{x} f^{-1}(y) \oplus f^{*} T_{f(x)} B=T_{x} A$, for any $x \in f^{-1}(y)$ where $f^{*}: T_{f(x)} B \rightarrow T_{x} A$ is a linear map satisfying $f_{*} \circ f^{*}=\operatorname{id}_{T_{f(x)} B}$. We denote by $-X$ the orientation reversed manifold of an oriented manifold $X$.

Let $X$ be a 3 -dimensional real vector space. Let $V$ be a finite set. Define $\breve{S}_{V}(X)$ to be the set of injective maps from $V$ to $X$ up to translations and dilations. Set $\underline{k}=\{1, \ldots, k\}$. We denote $\breve{S}_{\underline{k}}(X)$ by $\breve{S}_{k}(X)$. Note that $\breve{S}_{2}(X)=S(X)$. For an $\mathbb{R}^{3}$ vector bundle $E \rightarrow B$, we denote by $\breve{S}_{V}(E) \rightarrow B$ the fiber bundle where the fiber over $x \in B$ is $\breve{S}_{V}\left(E_{x}\right)$. 

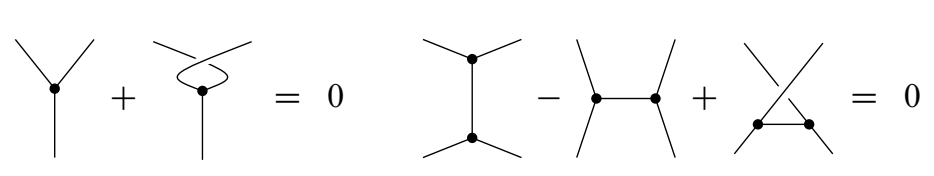

Figure 1: The relations AS (left) and IHX (right). Here the orientation of each vertex is given by counterclockwise order of the half edges.

\section{The Kontsevich-Kuperberg-Thurston invariant}

In this section, we introduce some notation about configuration spaces and Jacobi diagrams. Then we review the construction of the Kontsevich-Kuperberg-Thurston invariant. Most of this section follows [8].

\subsection{Jacobi diagrams}

This subsection follows [8, Sections 1.3, 2.3]. A Jacobi diagram of degree $n$ is defined to be a trivalent graph with $2 n$ vertices and $3 n$ edges without simple loops. For a Jacobi diagram $\bar{\Gamma}$, we denote by $H(\bar{\Gamma}), E(\bar{\Gamma})$ and $V(\bar{\Gamma})$ the set of half edges, the set of edges and the set of vertices, respectively. An orientation of a vertex of $\bar{\Gamma}$ is a cyclic order of the three half-edges that meet at the vertex. A Jacobi diagram is oriented if all its vertices are oriented. Let $\mathbb{R}\langle$ degree- $n$ oriented Jacobi diagrams $\rangle$ be the real vector space freely generated by all degree- $n$ oriented Jacobi diagrams. Let

$$
\mathcal{A}_{n}(\varnothing)=\mathbb{R}\langle\text { degree } n \text { oriented Jacobi diagrams }\rangle / \text { AS, IHX, }
$$

where the relations AS and IHX are locally represented as in Figure 1. Let

$$
\mathcal{E}_{n}=\left\{\Gamma=\left(\bar{\Gamma}, \varphi_{E}, \varphi_{V}, \text { ori }_{E}\right)\right\}
$$

up to isomorphism. Here $\bar{\Gamma}$ is a connected Jacobi diagram of degree $n, \varphi_{E}: E(\bar{\Gamma}) \cong$ $\{1,2, \ldots, 3 n\}$ and $\varphi_{V}: V(\bar{\Gamma}) \cong\{1,2, \ldots, 2 n\}$ are labels of edges and vertices respectively, and $\operatorname{ori}_{E}$ is a collection of orientations for all edges. For example, $\# \mathcal{E}_{1}=8$. These data and an orientation of $\bar{\Gamma}$ induce two orientations of $H(\bar{\Gamma})$. Here an orientation of a finite set is an order of all elements of the set up to even permutations. The first one is the edge-orientation induced by $\varphi_{E}$ and ori $E$. The edge-orientation is induced by the following order of $H(f)$ : the first half edge is the initial half-edge of $\varphi_{E}^{-1}(1)$ with respect to ori $E_{E}$, the second half edge is the terminal half-edge of $\varphi_{E}^{-1}(1)$, the third half edge is the initial half-edge of $\varphi_{E}^{-1}(2)$, and so on. The second one is the vertex-orientation induced by $\varphi_{V}$ and orientation of $\bar{\Gamma}$. The vertex-orientation is induced by the following order of $H(f)$ : the first three half edges meet at $\varphi_{V}^{-1}(1)$ with an order with respect to the orientation of $\bar{\Gamma}$, the second three half edges meet 
at $\varphi_{V}^{-1}(2)$, and so on. We choose the orientation of $\bar{\Gamma}$ so that the edge-orientation coincides with the vertex-orientation. Let us denote by $[\Gamma] \in \mathcal{A}_{n}(\varnothing)$ the oriented Jacobi diagram given by $\Gamma$ in such a way.

Remark 3.1 The notation $\mathcal{A}_{2 n, 3 n}$ used in [14] coincides with the notation $\mathcal{A}_{n}(\varnothing)$ used in [8] as $\mathbb{R}$-vector spaces.

\subsection{The configuration space $\breve{C}_{2 n}(Y)$}

This subsection follows [8, Sections 1.1, 1.2, 2.1].

Let $Y$ be a homology 3 -sphere with a basepoint $\infty$. Let $N(\infty ; Y)$ be a regular neighborhood (that is diffeomorphic to an open ball) of $\infty$ in $Y$, and let $N\left(\infty ; S^{3}\right)$ be a regular neighborhood of $\infty$ in $S^{3}=\mathbb{R}^{3} \cup \infty$. We fix an orientation-preserving diffeomorphism $\varphi^{\infty}:(N(\infty ; Y), \infty) \cong\left(N\left(\infty ; S^{3}\right), \infty\right)$ between $N(\infty ; Y)$ and $N\left(\infty ; S^{3}\right)$. We use $\varphi^{\infty}$ to identify $N(\infty ; Y)$ with $N\left(\infty ; S^{3}\right)$. Let $B_{Y}$ denote $Y \backslash N(\infty ; Y)$.

Let

$$
\breve{C}_{2 n}(Y)=(Y \backslash \infty)^{2 n} \backslash \Delta=\{\{1, \ldots, 2 n\} \hookrightarrow Y \backslash \infty\} .
$$

Let $C_{2}(Y)$ be given by real blowing up of $Y^{2}$ along $\infty^{2}=(\infty, \infty), \overline{\infty \times(Y \backslash \infty)}$, $\overline{(Y \backslash \infty) \times \infty}$ and $\overline{\Delta \backslash \infty^{2}}$ in turn, where the space $\overline{\infty \times(Y \backslash \infty)}$ is the closure of $\infty \times(Y \backslash \infty)$ in $B \ell\left(Y^{2}, \infty^{2}\right)$. Let us denote by $q: C_{2}(Y) \rightarrow Y^{2}$ the composition of the blowing down maps. Then

$$
\partial C_{2}(Y)=S T_{\infty} Y \times(Y \backslash \infty) \cup(Y \backslash \infty) \times S T_{\infty} Y \cup S v_{\Delta(Y \backslash \infty)} \cup q^{-1}\left(\infty^{2}\right) .
$$

The isomorphism

$$
\left(T Y^{2} \supset\right) v_{\Delta(Y \backslash \infty)} \rightarrow T(Y \backslash \infty), \quad(-u, u) \mapsto u,
$$

identifies $v_{\Delta(Y \backslash \infty)}$ with $T(Y \backslash \infty)$. Then we identify $S v_{\Delta(Y \backslash \infty)}$ with $\left.S T Y\right|_{Y \backslash \infty}$.

Let $p_{1}$ and $p_{2}$ denote the projections

$$
\begin{aligned}
& p_{1}:\left(\partial C_{2}(Y) \supset\right) S T_{\infty} Y \times(Y \backslash \infty) \rightarrow S T_{\infty} Y=S T_{\infty} S^{3}=S^{2}, \\
& p_{2}:\left(\partial C_{2}(Y) \supset\right)(Y \backslash \infty) \times S T_{\infty} Y \rightarrow S T_{\infty} Y=S T_{\infty} S^{3}=S^{2},
\end{aligned}
$$

where $S T_{\infty} Y$ and $S T_{\infty} S^{3}$ are identified by $\varphi^{\infty}$. We denote by ${ }^{\imath} S^{2}: S^{2} \rightarrow S^{2}$ the involution induced by $\times(-1): \mathbb{R}^{3} \rightarrow \mathbb{R}^{3}$.

From the definition of the compactification $C_{2}\left(S^{3}\right)$, it is possible to extend the map

$$
\text { int } C_{2}\left(S^{3}\right)=\left(\mathbb{R}^{3} \times \mathbb{R}^{3}\right) \backslash \Delta \rightarrow S^{2}, \quad(x, y) \mapsto(y-x) /\|y-x\|
$$


to $C_{2}\left(S^{3}\right)$ (see [8, Lemma 1.1] for more details). Let $p_{c}: C_{2}\left(S^{3}\right) \rightarrow S^{2}$ denote this map. Since $q^{-1}\left(N(\infty ; Y)^{2}\right) \subset C_{2}(Y)$ is identified with $q^{-1}\left(N\left(\infty ; S^{3}\right)^{2}\right) \subset C_{2}\left(S^{3}\right)$ by $\varphi^{\infty}$, we get a map $\partial C_{2}(Y) \cap q^{-1}\left(N(\infty ; Y)^{2}\right) \stackrel{p_{c}}{\longrightarrow} S^{2}$. Since $p_{1}, \iota_{S^{2}} \circ p_{2}$ and $p_{c}$ are compatible on the boundary, as in the case $Y=S^{3}$, these maps define the map

$$
p_{Y}: \partial C_{2}(Y) \backslash S T B_{Y} \rightarrow S^{2} .
$$

For each $\Gamma=\left(\bar{\Gamma}, \varphi_{E}, \varphi_{V}\right.$, ori $\left._{E}\right) \in \mathcal{E}_{n}$ and for each $\varphi_{E}^{-1}(i) \in E(\bar{\Gamma})$, let $s(i), t(i) \in$ $\{1, \ldots, 2 n\}$ denote the labels of the initial vertex and the terminal vertex of $\varphi_{E}^{-1}(i)$, respectively. The embedding $\{1,2\} \cong\{s(i), t(i)\} \hookrightarrow\{1, \ldots, 2 n\}$ induces the projection

$$
P_{i}(\Gamma)=\pi_{\breve{C}_{2 n}(Y)}: \breve{C}_{2 n}(Y) \rightarrow \breve{C}_{2}(Y)\left(\subset C_{2}(Y)\right) .
$$

We define a map $P_{i}(\Gamma): \breve{S}_{2 n}(E) \rightarrow S E$ in the same manner for an oriented $\mathbb{R}^{3}$ bundle $E \rightarrow B$. See [8, Section 2.3] for more details.

\subsection{Admissible forms and propagators}

Definition 3.2 (admissible form) A closed 2-form $\omega \in \Omega^{2}\left(\partial C_{2}(Y)\right)$ of $\partial C_{2}(Y)$ is an admissible form with respect to $\omega_{S^{2}}$ if the restriction of $\omega$ to $\partial C_{2}(Y) \backslash S T B_{Y}$ is $p_{Y}^{*} \omega_{S^{2}}$, where $\omega_{S^{2}}$ is a closed 2-form of $S^{2}$ with total volume 1 .

Let $\omega$ be an admissible form. Since $Y$ is a rational homology 3-sphere, we can check that $H^{2}\left(C_{2}(Y), \partial C_{2}(Y) ; \mathbb{R}\right) \cong H_{4}\left(C_{2}(Y) ; \mathbb{R}\right)=0$ and $H^{3}\left(C_{2}(Y), \partial C_{2}(Y) ; \mathbb{R}\right) \cong$ $H_{3}\left(C_{2}(Y) ; \mathbb{R}\right)=0$ as in the case $Y=S^{3}$ (see [8, Lemma 2.1] for more details). Then the restriction $H^{2}\left(C_{2}(Y) ; \mathbb{R}\right) \rightarrow H^{2}\left(\partial C_{2}(Y) ; \mathbb{R}\right)$ is an isomorphism. Thus there is a closed 2-form $\omega_{C}$ on $C_{2}(Y)$ satisfying $\left.\omega_{C}\right|_{\partial C_{2}(Y)}=\omega$.

Definition 3.3 (propagator) We call $\omega_{C}$ a propagator with respect to $\omega$.

Let $\left\{\omega_{i}\right\}_{i \in \underline{3 n}}$ be a collection of admissible forms.

Definition 3.4 Let $\omega_{i, C}$ be a propagator with respect to $\omega_{i}$. Then we define

$$
z_{n}\left(\left(\omega_{i}\right)_{i \in \underline{3 n}}\right)=\sum_{\Gamma \in \mathcal{E}_{n}}\left(\int_{\breve{C}_{2 n}(Y)} \bigwedge_{i \in \underline{3 n}} P_{i}(\Gamma)^{*} \omega_{i, C}\right)[\Gamma] \in \mathcal{A}_{n}(\varnothing) .
$$

We will see that this definition makes sense and is consistent in Lemma 3.5. For an $\mathbb{R}^{3}$-bundle $E$ over a manifold $Z$ with corners and a collection $\left(\eta_{i}\right)_{i \in \underline{3 n}}$ of forms over $S_{2}(E)$ satisfying $\sum_{i=1}^{3 n} \operatorname{deg}\left(\eta_{i}\right)=6 n-4+\operatorname{dim} Z$, define $X_{n}\left(E ;\left(\eta_{i}\right)_{\underline{3 n}}\right) \in \mathcal{A}_{n}(\varnothing)$ as

$$
X_{n}\left(E ;\left(\eta_{i}\right)_{\underline{3 n}}\right)=\sum_{\Gamma \in \mathcal{E}_{n}}\left(\int_{\breve{S}_{2 n}(E)} \bigwedge_{i \in \underline{3 n}} P_{i}(\Gamma)^{*} \eta_{i}\right)[\Gamma] .
$$

From the work of Lescop, especially in [8, Section 2.4], the following lemma holds. 
Lemma 3.5 (1) The integrals in Definition 3.4 are convergent.

(2) $z_{n}\left(\left(\omega_{i}\right)_{i \in \underline{3 n}}\right)$ is independent of the choice of propagators $\left(\omega_{i, C}\right)_{i \in \underline{3 n}}$.

(3) For any other collection $\left(\omega_{i}^{\prime}\right)_{i \in \underline{3 n}}$ of admissible forms, there exist closed 2-forms $\Omega_{i}=\Omega\left(\omega_{i}, \omega_{i}^{\prime}\right)$ on $[0,1] \times S T B_{Y}$ and closed 2 -forms $\Omega_{S^{2}, i}$ on $[0,1] \times S^{2}$ satisfying the following conditions:

(a) $\left.\Omega_{i}\right|_{\{0\} \times S T B_{Y}}=\left.\omega_{i}\right|_{S T B_{Y}}$,

(b) $\left.\Omega_{i}\right|_{\{1\} \times S T B_{Y}}=\left.\omega_{i}^{\prime}\right|_{S T B_{Y}}$,

(c) $\left.\Omega_{i}\right|_{[0,1] \times \partial S T B_{Y}}=\left(\mathrm{id} \times p_{Y}\right)^{*} \Omega_{S^{2}, i}$,

(d) $\left.\Omega_{S^{2}, i}\right|_{\{0\} \times S^{2}}=\omega_{S^{2}, i}$ and

(e) $\left.\Omega_{S^{2}, i}\right|_{\{1\} \times S^{2}}=\omega_{S^{2}, i}^{\prime}$.

Here $\left.\omega_{i}\right|_{\partial C_{2}(Y) \backslash S T B_{Y}}=p_{Y}^{*} \omega_{S^{2}, i}$ and $\left.\omega_{i}^{\prime}\right|_{\partial C_{2}(Y) \backslash S T B_{Y}}=p_{Y}^{*} \omega_{S^{2}, i}^{\prime}$. For these forms, $z_{n}\left(\left(\omega_{i}^{\prime}\right)_{i \in \underline{3 n}}\right)-z_{n}\left(\left(\omega_{i}\right)_{i \in \underline{3 n}}\right)=X_{n}\left([0,1] \times T B_{Y} ;\left(\Omega_{i}\right)_{i \in \underline{3 n}}\right)$.

The proof of this lemma will be outlined in Section 5.1.

\subsection{Review of the Kontsevich-Kuperberg-Thurston invariant}

In this section, we review the construction of the Kontsevich-Kuperberg-Thurston invariant $z_{n}^{\mathrm{KKT}}$ for rational homology 3-spheres with a little modification.

For a framing $\tau^{\prime}$ of $T Y$, we denote by $\sigma_{Y}\left(\tau^{\prime}\right) \in \mathbb{Z}$ the signature defect of $\tau^{\prime}$ defined as follows: Take a compact $4-$ manifold $X_{0}$ bounded by $Y$. Let $p_{1}\left(\tau^{\prime} ; X_{0}\right) \in$ $H^{4}\left(X_{0}, Y ; \mathbb{Z}\right)$ be the relative Pontrjagin class. By the Hirzebruch signature theorem, the difference $\sigma_{Y}\left(\tau^{\prime}\right)=\int_{X_{0}} p_{1}\left(\tau^{\prime} ; X_{0}\right)-3 \operatorname{Sign} X_{0}$ is independent of the choice of $X_{0}$. See [5] or [1] for more details. Let $\tau_{S^{3}}$ be a framing ${ }^{1}$ of $T S^{3}$ satisfying the following two conditions:

- $\sigma_{S^{3}}\left(\tau_{S^{3}}\right)=2$,

- $\left.\tau_{S^{3}}\right|_{S^{3} \backslash N^{\prime}\left(\infty ; S^{3}\right)}=\left.\tau_{\mathbb{R}^{3}}\right|_{S^{3} \backslash N^{\prime}\left(\infty ; S^{3}\right)}$, where $\tau_{\mathbb{R}^{3}}: T \mathbb{R}^{3} \cong \underline{\mathbb{R}^{3}}$ is the standard framing of $T \mathbb{R}^{3}$.

Here $N^{\prime}\left(\infty ; S^{3}\right)$ is a neighborhood of $\infty$ smaller than $N\left(\infty ; S^{3}\right)$; that is, $\infty \in$ $N^{\prime}\left(\infty ; S^{3}\right) \subset N\left(\infty ; S^{3}\right)$.

Remark 3.6 There is no special meaning in the number 2 in the first condition above. The anomaly term $\widetilde{z}_{n}^{\text {anomaly }}(\vec{\gamma})$ would be independent of the choice of $\tau_{S^{3}}$ even if $\sigma_{S^{3}}\left(\tau_{S^{3}}\right)$ were not 2 . We remark that there is no framing $\tau$ on $S^{3}$ such that $\sigma_{S^{3}}(\tau)=0$.

${ }^{1}$ There is such a framing. For example, the Lie framing $\tau_{\mathrm{SU}(2)}$ of the tangent bundle of $S^{3}=\mathrm{SU}(2)$ corresponding to left multiplication satisfies $\sigma_{S^{3}}\left(\tau_{\mathrm{SU}(2)}\right)=2$. See [5] for more details. We can get $\tau_{S^{3}}$ by modifying $\tau_{\mathrm{SU}(2)}$. 
Let $\tau_{Y}: T(Y \backslash \infty) \cong \underline{\mathbb{R}^{3}}$ be a framing satisfying $\left.\tau_{Y}\right|_{N(\infty ; Y) \backslash \infty}=\tau_{\mathbb{R}^{3}}$, where $\underline{\mathbb{R}^{3}}$ is the trivial $\mathbb{R}^{3}$-bundle over $N(\infty ; Y) \backslash \infty$. We call such a framing an admissible framing. We have that $\left.\left.\tau_{Y}\right|_{Y \backslash N(\infty ; Y)} \cup \tau_{S^{3}}\right|_{N\left(\infty ; S^{3}\right)}$ is a framing of $T Y$ by the above conditions on $\tau_{Y}$. We define

$$
\begin{aligned}
\sigma_{Y \backslash \infty}\left(\tau_{Y}\right) & =\sigma_{Y}\left(\left.\left.\tau_{Y}\right|_{Y \backslash N(\infty ; Y)} \cup \tau_{S^{3}}\right|_{N\left(\infty ; S^{3}\right)}\right)-\sigma_{S^{3}}\left(\tau_{S^{3}}\right) \\
& =\sigma_{Y}\left(\left.\left.\tau_{Y}\right|_{Y \backslash N(\infty ; Y)} \cup \tau_{S^{3}}\right|_{N\left(\infty ; S^{3}\right)}\right)-2,
\end{aligned}
$$

and call it the signature defect of $\tau_{Y}$. For example, $\sigma_{\mathbb{R}^{3}}\left(\tau_{\mathbb{R}^{3}}\right)=0$.

The composition of the framing $\tau_{Y}$ and the projection map $\underline{\mathbb{R}^{3}} \rightarrow \mathbb{R}^{3}$ induces the map $p_{\Delta}\left(\tau_{Y}\right): S T B_{Y} \rightarrow S^{2}$. Thanks to the conditions we imposed on $\tau_{Y}$, the maps $p_{\Delta}\left(\tau_{Y}\right)$ and $p_{Y}: \partial C_{2}(Y) \backslash S T B_{Y} \rightarrow S^{2}$ are compatible. So we get the map

$$
p\left(\tau_{Y}\right)=p_{Y} \cup p_{\Delta}\left(\tau_{Y}\right): \partial C_{2}(Y) \rightarrow S^{2} .
$$

Therefore, we have an admissible form $\omega_{\tau_{Y}}=p\left(\tau_{Y}\right)^{*} \omega_{S^{2}}$ for any closed 2-form $\omega_{S^{2}}$ of $S^{2}$ with total volume 1 .

Definition 3.7 We define $z_{n}^{\mathrm{KKT}}\left(Y ; \tau_{Y}\right)=z_{n}\left(\left(\omega_{\tau_{Y}}\right)_{i \in \underline{3 n}}\right)$.

Lemma 3.8 [8, Theorem 1.9, Proposition 2.11] For any other framing $\tau_{Y}^{\prime}$, there exist constants $\delta_{n} \in \mathcal{A}_{n}(\varnothing)$ such that

$$
z_{n}^{\mathrm{KKT}}\left(Y ; \tau_{Y}^{\prime}\right)-z_{n}^{\mathrm{KKT}}\left(Y ; \tau_{Y}\right)=-\frac{1}{4}\left(\sigma_{Y \backslash \infty}\left(\tau_{Y}^{\prime}\right)-\sigma_{Y \backslash \infty}\left(\tau_{Y}\right)\right) \delta_{n} .
$$

The central definition that we study here generalizes the definitions that are given in [7] by Kuperberg and Thurston and in [8] by Lescop.

Definition 3.9 Let $\left(\omega_{i}\right)_{i \in \underline{3 n}}$ be a collection of admissible forms and $\tau_{Y}$ be an admissible framing. We define

where

$$
z_{n}^{\mathrm{KKT}}(Y)=z_{n}\left(\left(\omega_{i}\right)_{i \in \underline{3 n}}\right)-\widetilde{z}_{n}^{\text {anomaly }}\left(\left(\omega_{i}\right)_{i \in \underline{3 n}}\right) \in \mathcal{A}_{n}(\varnothing),
$$

$$
\widetilde{z}_{n}^{\text {anomaly }}\left(\left(\omega_{i}\right)_{i \in \underline{3 n}}\right)=X_{n}\left([0,1] \times T B_{Y} ;\left(\Omega_{i}\left(\omega_{\tau_{Y}}, \omega_{i}\right)\right)_{i \in \underline{3 n}}\right)-\frac{1}{4} \sigma_{Y \backslash \infty}\left(\tau_{Y}\right) \delta_{n} .
$$

Lemmas 3.5 and 3.8 imply that Definition 3.9 is consistent.

We will give an alternative description of $\widetilde{z}_{n}^{\text {anomaly }}\left(\left(\omega_{i}\right)_{i \in \underline{3 n}}\right)$ without framings in Section 4.1. We remark that $\delta_{n}$ is given by an explicit formula in [8, Proposition 2.10]. Lemma 3.5(3) implies that $\widetilde{z}_{n}^{\text {anomaly }}\left(\left(\omega_{i}\right)_{i \in \underline{3 n}}\right)$ is independent of the choice of $\tau_{Y}$. Thus $\widetilde{z}_{n}^{\text {anomaly }}\left(\left(\omega_{i}\right)_{i \in \underline{3 n}}\right)$ is consistent. 
Remark 3.10 The universal finite type invariant $Z_{n}^{\mathrm{KKT}}$ described in [8] is equal to the degree- $n$ part of

$$
\exp \left(\sum_{n} \frac{1}{2^{3 n}(3 n) !(2 n) !} z_{n}^{\mathrm{KKT}}\right) .
$$

See the discussion before Lemma 2.12 in [8] for more details.

\section{Alternative description of $z_{n}^{\mathrm{KKT}}$}

This section is the main part of this paper. In Section 4.1, we give an alternative description of the anomaly term. In Section 4.2, we give a construction of an admissible form by using a vector field. In Section 4.3, we give an explicit propagator by using a Morse function. This construction is close to T Watanabe's Morse homotopy invariant.

\subsection{Alternative description of the anomaly term $\widetilde{z}_{n}^{\text {anomaly }}\left(\left(\omega_{i}\right)_{i \in \underline{3 n}}\right)$}

The idea of this alternative construction is based on the construction of the anomaly term of Watanabe's invariant in [14]. We note that Watanabe defined his invariant only for integral homology 3-spheres.

Let $Y$ be a closed 3-manifold with a basepoint $\infty \in Y$. Let $\omega_{i}$ be an admissible form with respect to a closed 2-form $\omega_{S^{2}, i}$ of $S^{2}$. Let $\tau_{S^{3}}$ be a framing as in Section 3.4. The framing $\tau_{S^{3}}$ induces the map $p\left(\tau_{S^{3}}\right): S T S^{3} \rightarrow S^{2}$.

Let $X$ be a compact connected 4-manifold with $\partial X=Y$ and $\chi(X)=0$. For example, we can take $X=\left(T^{4} \# \mathbb{C} P^{2}\right) \backslash B^{4}$ when $Y=S^{3}$. Since $\chi(X)=0$, it is possible to extend $T Y$ to an oriented $\mathbb{R}^{3}$-subbundle of $T X$. We denote by $T^{v} X$ such an extended bundle. Let $F_{X} \rightarrow S T^{v} X$ be the tangent bundle along the fiber of the sphere bundle $S T^{v} X \rightarrow X$. Let $W_{i}$ be a closed 2 -form on $S T^{v} X$ satisfying the following conditions:

- $2 W_{i}$ represents the Euler class $e\left(F_{X}\right)$ of $F_{X} \rightarrow S T^{v} X$,

- $\left.W_{i}\right|_{S T Y}=\left.\left.\omega_{i}\right|_{S T B_{Y}} \cup p\left(\tau_{S^{3}}\right)^{*} \omega_{S^{2}, i}\right|_{S T N(\infty ; Y)}$.

Theorem 4.1 $\widetilde{z}_{n}^{\text {anomaly }}\left(\left(\omega_{i}\right)_{i \in \underline{3 n}}\right)=\frac{3}{4} \delta_{n} \operatorname{Sign} X+X_{n}\left(T^{v} X ;\left(W_{i}\right)_{i \in \underline{3 n}}\right)+\frac{1}{2} \delta_{n} \in \mathcal{A}_{n}(\varnothing)$.

For any closed connected 4-manifold $X^{\prime}$ with $\chi\left(X^{\prime}\right)=0$, we can define $T^{v} X^{\prime}$ and $X\left(T^{v} X^{\prime} ;\left(W_{i}^{\prime}\right)_{i \in \underline{3 n}}\right)$ for any closed 2-form $W_{i}^{\prime}$ of $S T^{v} X^{\prime}$ which represents $\frac{1}{2} e\left(F_{X^{\prime}}\right)$. The well-definedness of the right hand side of Theorem 4.1 will be a direct consequence of the following theorem. 
Theorem 4.2 For any closed connected 4-manifold $X^{\prime}$ with $\chi\left(X^{\prime}\right)=0$,

$$
-\frac{3}{4} \delta_{n} \operatorname{Sign} X^{\prime}=X_{n}\left(T^{v} X^{\prime} ;\left(W_{i}^{\prime}\right)_{i \in \underline{3 n}}\right) .
$$

Theorem 4.1 and 4.2 will be proved in Section 5.

\subsection{An admissible form given by a collection of vector fields}

In this section, we give a construction of an admissible form by using a smooth vector field. We will use these admissible forms in the next subsection.

For $a \in S^{2} \subset \mathbb{R}^{3}$, the map $q_{a}: \mathbb{R}^{3} \rightarrow \mathbb{R}$ is defined by $q_{a}(x)=\langle x, a\rangle$, where $\langle$,$\rangle is$ the standard inner product on $\mathbb{R}^{3}$.

Definition 4.3 A smooth vector field $\gamma$ on $Y \backslash \infty$ is an admissible vector field (with respect to $a$ ) if the following conditions hold:

- $\left.\gamma\right|_{N(\infty ; Y) \backslash \infty}=a\left(=\left.\operatorname{grad} q_{a}\right|_{N\left(\infty ; S^{3}\right) \backslash \infty}\right)$,

- $\gamma$ is transverse to the zero section in $T(Y \backslash \infty)$.

Example 4.4 We give two important examples of admissible vector fields with respect to $a$ :

(1) Let $\tau_{\mathbb{R}^{3}}: T \mathbb{R}^{3} \cong \underline{\mathbb{R}^{3}}$ be the standard framing of $T \mathbb{R}^{3}$. We regard $a \in \mathbb{R}^{3}$ as a constant section of the trivial bundle $\underline{\mathbb{R}}^{3}$. If $\tau: T(Y \backslash \infty) \cong \underline{\mathbb{R}^{3}}$ is an admissible framing, the pullback vector field $\tau^{*} a$ is an admissible vector field with respect to $a$.

(2) For a Morse function $f: Y \backslash \infty \rightarrow \mathbb{R}$ such that $\left.f\right|_{N(\infty ; Y) \backslash \infty}=\left.q_{a}\right|_{N(\infty ; S) \backslash \infty}$, $\operatorname{grad} f$ is an admissible vector field with respect to $a$.

The following lemma plays an important role in this construction. For an admissible vector field $\gamma$, let

$$
\bar{c}_{\gamma}=\overline{\left\{[\gamma(x)] \in S T_{x} Y \mid x \in Y \backslash\left(\infty \cup \gamma^{-1}(0)\right)\right\}} \subset S T(Y \backslash \infty),
$$

where $[\gamma(x)] \in S T_{x} Y$ is the class of $\gamma(x) \in T_{x} Y$ up to dilation. Here we choose the orientation of $\bar{c}_{\gamma}$ such that the restriction to $\bar{c}_{\gamma}$ of the projection $S T Y \rightarrow Y$ is orientation preserving.

Lemma $4.5 c_{0}(\gamma)=\bar{c}_{\gamma} \cup \bar{c}_{-\gamma}$ is a submanifold of $S T(Y \backslash \infty)$ without boundary. 
Proof It is sufficient to check this claim near $\gamma^{-1}(0)$. Let $x \in \gamma^{-1}(0)$. Identify a neighborhood of $x$ with an open neighborhood $U$ of 0 in $\mathbb{R}^{3}$ by a chart that maps $x$ to 0 . Then $\gamma(u)$ reads $\left(u \in U, \Psi(u) \in T_{u} \mathbb{R}^{3}=\mathbb{R}^{3}\right)$ in such a chart where $\Psi(0)=0$ and $d_{0} \Psi \in G L\left(T_{0} \mathbb{R}^{3}\right)=G L\left(\mathbb{R}^{3}\right)$. Under this chart,

$$
\left.c_{\gamma} \cap S T Y\right|_{U}=\left\{\left(t v, \frac{\Psi(t v)}{\|\Psi(t v)\|}\right) \mid t \in(0, \infty), v \in S^{2}, t v \in U\right\} .
$$

Set $\psi_{t}(v)=\Psi(t v) /\|\Psi(t v)\|$ when $t \in(0, \infty)$ and $\psi_{0}(v)=d_{0} \Psi(v) /\left\|d_{0} \Psi(v)\right\|$ for $v \in S^{2}$. Thus $\psi_{0}(v)$ is the limit of $\psi_{t}(v)=\Psi(t v) /(t\|(1 / t) \Psi(t v)\|)$ where $t$ approaches 0 . Then

$$
\begin{gathered}
\left.\bar{c}_{\gamma} \cap S T Y\right|_{U}=\left\{\left(t v, \psi_{t}(v)\right) \mid t \in[0, \infty), v \in S^{2}, t v \in U\right\}, \\
\left.\bar{c}_{-\gamma} \cap S T Y\right|_{U}=\left\{\left(t v,-\psi_{t}(v)\right) \mid t \in[0, \infty), v \in S^{2}, t v \in U\right\} .
\end{gathered}
$$

Let $\varepsilon_{0} \in\{1,-1\}$ be the sign of the Jacobian of $\psi_{0}$; namely $\varepsilon_{0}=1$ if and only if the Jacobian of $\psi_{0}$ is positive. Fix $\varepsilon>0$ small enough so that for any $t \in[0, \varepsilon)$, we have $t S^{2} \subset U$ and $\psi_{t}$ is a diffeomorphism of $S^{2}$. Let $B(\varepsilon)=\left\{x \in \mathbb{R}^{3} \mid\|x\|<\varepsilon\right\}$. We have $\left.c_{0}(\gamma) \cap S T Y\right|_{B(\varepsilon)}$ $=\varepsilon_{0}\left\{\left(t \psi_{t}^{-1}(w), w\right) \mid t \in[0, \varepsilon), w \in S^{2}\right\} \cup-\varepsilon_{0}\left\{\left(t \psi_{t}^{-1}(-w), w\right) \mid t \in[0, \varepsilon), w \in S^{2}\right\}$. Let $\Phi(t, w)=\left(|t| \psi_{|t|}^{-1}((t /|t|) w), w\right)$ if $t \neq 0$ and $\Phi(0, w)=(0, w)$. Then $\Phi$ is a smooth embedding. Furthermore, $\left.\Phi\right|_{(0, \varepsilon) \times S^{2}}$ (resp. $\left.\Phi\right|_{(-\varepsilon, 0) \times S^{2}}$ ) is an embedding into $c_{\gamma}$ (resp. $c_{-\gamma}$ ) that preserves the orientation if and only if $\psi_{0}$ does, so that

$$
\left.c_{0}(\gamma) \cap S T Y\right|_{B(\varepsilon)}=\left\{\Phi(t, w) \mid t \in(-\varepsilon, \varepsilon), w \in S^{2}\right\} .
$$

Then $c_{0}(\gamma)$ is an oriented topological manifold that reads as the image of the embedding $\Phi$ near $\left.S T Y\right|_{x}$.

We define

$$
c(\gamma)=p_{Y}^{-1}(a) \cup p_{Y}^{-1}(-a) \cup\left(c_{0}(\gamma) \cap S T B_{Y}\right) \subset \partial C_{2}(Y) .
$$

By the definition of admissible vector fields and by Lemma 4.5, $c(\gamma)$ is a closed 3 -manifold. Therefore, $c(\gamma)$ determines a 3-cycle of $\partial C_{2}(Y)$.

Let $\omega_{S^{2}}^{a}$ be an antisymmetric closed 2-form on $S^{2}$ such that $\omega_{S^{2}}^{a}$ represents $\frac{1}{2}$ times the Poincaré dual of $[a]+[-a]$ and the support of $\omega_{S^{2}}^{a}$ is concentrated near $\{a,-a\}$. Let $\omega(\gamma)$ be an admissible form on $\partial C_{2}(Y)$ satisfying the following conditions: ${ }^{2}$

\footnotetext{
${ }^{2}$ All admissible forms satisfy the first condition because $H^{2}\left(\partial C_{2}(Y) ; \mathbb{R}\right) \cong \mathbb{R}$.
} 
- $2 \omega(\gamma)$ represents the Poincaré dual of $[c(\gamma)]$,

- the support of $\omega(\gamma)$ is concentrated near $c(\gamma)$, and

- $\left.\omega(\gamma)\right|_{\partial C_{2}(Y) \backslash S T B_{Y}}=p_{Y}^{*} \omega_{S^{2}}^{a}$; namely $\omega(\gamma)$ is an admissible form.

\subsection{Morse homotopy theoretic description of $z_{n}^{\mathrm{KKT}}$}

In this subsection, we give a Morse homotopy theoretic description of $z_{n}^{\mathrm{KKT}}$ by using a collection of $3 n$ Morse functions. This description is deeply inspired by the work of Watanabe in [14].

We first prepare some notations about Morse functions. Let $Y$ be a closed 3-dimensional manifold with a basepoint $\infty \in Y$. Fix a point $a \in S^{2}$.

Definition 4.6 A Morse function $f: Y \backslash \infty \rightarrow \mathbb{R}$ is an admissible Morse function with respect to $a$ if it satisfies the following conditions:

- $\left.f\right|_{N(\infty ; Y) \backslash \infty}=\left.q_{a}\right|_{N\left(\infty ; S^{3}\right) \backslash \infty}$, and

- $f$ has no critical point of index 0 or 3 .

Let $f$ be a Morse function on $Y \backslash \infty$ (or $Y$ ) and $\xi$ a metric on $Y$ satisfying the MorseSmale condition. Let grad $f$ be the gradient vector field of $f$ corresponding to $\xi$. Let Crit $(f)$ denote the set of all critical points of $f$. Let $\left\{\Phi_{f}^{t}\right\}_{t \in \mathbb{R}}: Y \backslash \infty \rightarrow Y \backslash \infty$ be the 1-parameter group of diffeomorphisms associated to $\operatorname{grad} f$. We denote by

$$
\mathcal{A}_{p}=\left\{x \in Y \mid \lim _{t \rightarrow-\infty} \Phi_{f}^{t}(x)=p\right\} \quad \text { and } \quad \mathcal{D}_{p}=\left\{x \in Y \mid \lim _{t \rightarrow \infty} \Phi_{f}^{t}(x)=p\right\}
$$

the ascending manifold and the descending manifold at $p \in \operatorname{Crit}(f)$, respectively.

Let us orient ascending manifolds and descending manifolds by imposing the condition $T_{p} \mathcal{A}_{p} \oplus T_{p} \mathcal{D}_{p} \cong T_{p} Y$ for any $p \in \operatorname{Crit}(f)$. Let $p, q \in \operatorname{Crit}(f)$ be critical points of indices 2 and 1, respectively. By the Morse-Smale condition, $\mathcal{D}_{p} \cap \mathcal{A}_{q}$ is a 1 -manifold. Let us orient $\mathcal{D}_{p} \cap \mathcal{A}_{q}$ by the following rule:

We fix a Riemannian metric on $Y$. The normal bundle $N \mathcal{D}_{p}$ of $\mathcal{D}_{p}$ and the normal bundle $N \mathcal{A}_{q}$ of $\mathcal{A}_{q}$ are oriented by

$$
T \mathcal{D}_{p} \oplus N \mathcal{D}_{p}=\left.T Y\right|_{\mathcal{D}_{p}} \quad \text { and } \quad T \mathcal{A}_{q} \oplus N \mathcal{A}_{q}=\left.T Y\right|_{\mathcal{A}_{q}}
$$

Let $n\left(\mathcal{D}_{p}\right)$ and $n\left(\mathcal{A}_{q}\right)$ be positively oriented normal vector fields of $\mathcal{D}_{p}$ and $\mathcal{A}_{q}$, respectively. We orient the normal bundle $N\left(\mathcal{D}_{p} \cap \mathcal{A}_{q}\right)$ of $\mathcal{D}_{p} \cap \mathcal{A}_{q}$ by using the frame $\left(\left.n\left(\mathcal{A}_{q}\right)\right|_{\mathcal{D}_{p} \cap \mathcal{A}_{q}},\left.n\left(\mathcal{D}_{p}\right)\right|_{\mathcal{D}_{p} \cap \mathcal{A}_{q}}\right)$. Then we orient $\mathcal{D}_{p} \cap \mathcal{A}_{q}$ by

$$
T\left(\mathcal{D}_{p} \cap \mathcal{A}_{q}\right) \oplus N\left(\mathcal{D}_{p} \cap \mathcal{A}_{q}\right)=\left.T Y\right|_{\mathcal{D}_{p} \cap \mathcal{A}_{q}} .
$$




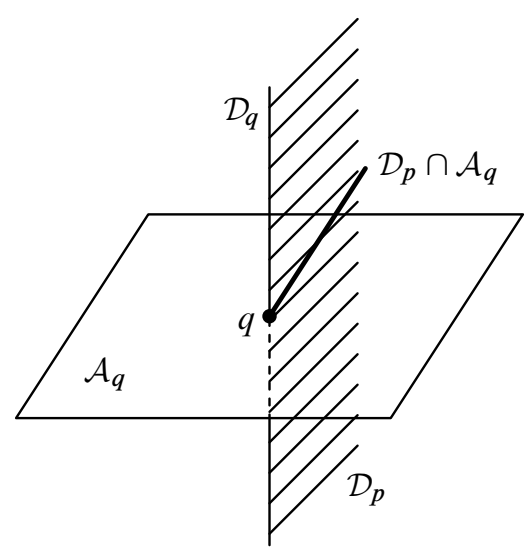

Figure 2: The orientation of $\mathcal{D}_{p} \cap \mathcal{A}_{q}$

We give a sign \pm 1 to each connected component of $\mathcal{D}_{p} \cap \mathcal{A}_{q}$. A component has sign +1 if the orientation of this component is from $q$ to $p$. See Figure 2 .

We assume $f$ is an admissible Morse function. Let $\operatorname{Crit}(f)=\left\{p_{1}, \ldots, p_{k}, q_{1}, \ldots, q_{k}\right\}$ be the set of critical points of $f$ where $\operatorname{ind}\left(p_{i}\right)=2$ and $\operatorname{ind}\left(q_{i}\right)=1$. Let

$$
0 \rightarrow C_{2}(Y \backslash \infty ; f) \stackrel{\widehat{\partial}}{\rightarrow} C_{1}(Y \backslash \infty ; f) \rightarrow 0
$$

be the Morse complex of $f$ with rational coefficients. Here the boundary map is defined as $\hat{\partial}\left(\left[p_{i}\right]\right)=\sum_{j} \widehat{\partial}_{i j}\left[q_{j}\right]$, where $\widehat{\partial}_{i j}$ is the sum of the signs of all the connected components of $\mathcal{D}_{p_{i}} \cap \mathcal{A}_{q_{j}}$. Let

$$
\hat{g}: C_{1}(Y \backslash \infty ; f) \rightarrow C_{2}(Y \backslash \infty ; f), \quad \hat{g}\left(\left[q_{j}\right]\right)=\sum_{j} \hat{g}_{j i}\left[p_{i}\right],
$$

be the inverse map of $\widehat{\partial}$. (In [14], $\widehat{g}$ is called a combinatorial propagator.)

We now construct $\mathcal{M}(f)$, which is a weighted sum of (noncompact) 4-manifolds in $Y^{2} \backslash \Delta$. Let $M_{\rightarrow}(f)$ be the 4-manifold which is the image of the inclusion map

$$
\varphi_{f}:(Y \backslash \infty) \times(0, \infty) \hookrightarrow(Y \backslash \infty) \times(Y \backslash \infty), \quad(x, t) \mapsto\left(x, \Phi_{f}^{t}(x)\right) .
$$

We choose the orientation of $M_{\rightarrow}(f)$ so that the map $\varphi_{f}$ preserves orientations. Define

$$
\mathcal{M}(f)=M_{\rightarrow}(f)+\sum_{i, j} \hat{g}_{j i}\left(\mathcal{D}_{p_{i}} \times \mathcal{A}_{q_{j}}\right) \backslash \Delta .
$$

We remark that the orientation of $\mathcal{M}(f)$ does not depend on the choice of orientations of $\mathcal{D}_{p_{i}}$ and $\mathcal{A}_{q_{j}}$. 
Let $a_{1}, \ldots, a_{3 n} \in S^{2} \subset \mathbb{R}^{3}$ be points such that any three distinct points are linearly independent in $\mathbb{R}^{3}$. Let $f_{1}, \ldots, f_{3 n}$ be a collection of sufficiently generic admissible Morse functions with respect to $a_{1}, \ldots, a_{3 n}$. Take metrics $\xi_{1}, \ldots, \xi_{3 n}$ on $Y$ and use $\xi_{i}$ for $\operatorname{grad} f_{i}$.

Set $\mathcal{M}\left( \pm f_{i}\right)=\mathcal{M}\left(f_{i}\right)+\mathcal{M}\left(-f_{i}\right)$. For generic $\xi_{i}, f_{i}$ and $a_{i}$, the weighted sums of manifolds

$$
\left.P_{1}(\Gamma)\right|_{(Y \backslash \infty)^{2 n} \backslash \Delta} ^{-1}\left(\mathcal{M}\left( \pm f_{1}\right)\right), \ldots,\left.P_{3 n}(\Gamma)\right|_{(Y \backslash \infty)^{2 n} \backslash \Delta} ^{-1}\left(\mathcal{M}\left( \pm f_{3 n}\right)\right)
$$

transversally intersect at finitely many points for any $\Gamma \in \mathcal{E}_{n}$. We can prove this fact in a similar manner as in the proofs of Proposition 2.4 and Lemma 4.2 in [14].

Theorem 4.7 For generic $f_{1}, \ldots, f_{3 n}$ and $\xi_{1}, \ldots, \xi_{3 n}$, $z_{n}^{\mathrm{KKT}}(Y)$

$$
=\sum_{\Gamma \in \mathcal{E}_{n}} \frac{1}{2^{3 n}} \#\left(\left.\bigcap_{i=1}^{3 n} P_{i}(\Gamma)\right|_{(Y \backslash \infty)^{2 n} \backslash \Delta} ^{-1}\left(\mathcal{M}\left( \pm f_{i}\right)\right)\right)[\Gamma]-\widetilde{z}_{n}^{\text {anomaly }}\left(\left(\omega\left(\operatorname{grad} f_{i}\right)\right)_{i \in \underline{3 n}}\right) .
$$

Here $\omega\left(\operatorname{grad} f_{i}\right)$ is an admissible form corresponding to the admissible vector field $\operatorname{grad} f_{i}$.

\section{Proofs}

In this section we prove Theorems 4.1, 4.2 and 4.7.

\subsection{Needed lemmas from [8]}

We first give the outline of the proof of Lemma 3.5. See [8, Section 2.4] for more details.

\section{Outline of the proof of Lemma 3.5}

(1) See [8, Proposition 2.5].

(2) See [8, Proposition 2.9].

(3) Fix $j \in \underline{3 n}$. It is enough to study the case where $\omega_{i}=\omega_{i}^{\prime}$ for any $i \neq j$. Since $\left[\omega_{j}^{\prime}\right]=\left[\omega_{j}\right] \in H^{2}\left(\partial C_{2}(Y) ; \mathbb{R}\right)$ and $\left[\omega_{S^{2}, j}^{\prime}\right]=\left[\omega_{S^{2}, j}\right] \in H^{2}\left(S^{2} ; \mathbb{R}\right)$, there exist closed $2-$ forms $\Omega_{j}^{0}$ on $[0,1] \times \partial C_{2}(Y)$ and $\Omega_{S^{2}, j}$ on $[0,1] \times S^{2}$ such that

- $\left.\Omega_{j}^{0}\right|_{\{0\} \times \partial C_{2}(Y)}=\omega_{j}$,

- $\left.\Omega_{j}^{0}\right|_{\{1\} \times \partial C_{2}(Y)}=\omega_{j}^{\prime}$,

- $\left.\Omega_{S^{2}, j}\right|_{\{0\} \times S^{2}}=\omega_{S^{2}, j}$ and

- $\left.\Omega_{S^{2}, j}\right|_{\{1\} \times S^{2}}=\omega_{S^{2}, j}^{\prime}$. 
More precisely, by the same argument of Lemma 2.15 in [8], we can take $\Omega_{j}^{0}$ so that $\left.\Omega_{j}^{0}\right|_{[0,1] \times\left(\partial C_{2}(Y) \backslash S T B_{Y}\right)}=p_{Y}^{*} \Omega_{S^{2}, j}$. Let $\Omega_{i}^{0}=\pi^{*} \omega_{i}$ for any $i \neq j$, where $\pi:[0,1] \times \partial C_{2}(Y) \rightarrow \partial C_{2}(Y)$ is the projection. Similarly, let $\Omega_{S^{2}, i}=\pi^{*} \omega_{S^{2}, i}$ for $i \neq j$, where $\pi:[0,1] \times S^{2} \rightarrow S^{2}$ is the projection. Set $\Omega_{i}=\left.\Omega_{i}^{0}\right|_{[0,1] \times S T B_{Y}}$.

On the other hand, there is a natural compactification $C_{2 n}(Y)$ of $\breve{C}_{2 n}(Y)$; this is a compact $6 n$-dimensional manifold with corners, and there is a natural extension to $C_{2 n}(Y)$ of $P_{i}(\Gamma): \breve{C}_{2 n}(Y) \rightarrow C_{2}(Y)$; see [8, Section 3] for more details. By Stokes' theorem,

$$
\begin{aligned}
0 & =\sum_{\Gamma \in \mathcal{E}_{n}} \int_{[0,1] \times C_{2 n}(Y)} d\left(\bigwedge_{i}\left(\mathrm{id} \times P_{i}(\Gamma)\right)^{*} \Omega_{i}^{0}\right)[\Gamma] \\
& =z_{n}\left(\left(\omega_{i}^{\prime}\right)_{i \in \underline{3 n}}\right)-z_{n}\left(\left(\omega_{i}\right)_{i \in \underline{3 n}}\right)-\sum_{\Gamma \in \mathcal{E}_{n}} \int_{[0,1] \times \partial C_{2 n}(Y)}\left(\bigwedge_{i}\left(\mathrm{id} \times P_{i}(\Gamma)\right)^{*} \Omega_{i}^{0}\right)[\Gamma] .
\end{aligned}
$$

Lemmas $2.17,2,18,2.19,2.20$ and 2.21 of [8] imply that

$$
\begin{aligned}
\sum_{\Gamma \in \mathcal{E}_{n}} \int_{[0,1] \times \partial C_{2 n}(Y)}\left(\bigwedge_{i}\left(\mathrm{id} \times P_{i}(\Gamma)\right)^{*} \Omega_{i}^{0}\right)[\Gamma] & \\
& =\sum_{\Gamma \in \mathcal{E}_{n}} \int_{[0,1] \times \breve{S}_{2 n}\left(T B_{Y}\right)}\left(\bigwedge_{i}\left(\mathrm{id} \times P_{i}(\Gamma)\right)^{*} \Omega_{i}\right)[\Gamma] \\
& =X_{n}\left([0,1] \times T B_{Y} ;\left(\Omega_{i}\right)_{i \in \underline{3 n}}\right) .
\end{aligned}
$$

Two lemmas To prove Theorem 4.1 and Theorem 4.2, we prepare two lemmas. These lemmas are essentially given by C Lescop in [8].

Lemma 5.1 Let $E \rightarrow Z$ be an oriented $\mathbb{R}^{3}$-bundle over a manifold $Z$ with corners and let $\left(\eta_{i}\right)_{i \in \underline{3 n}}$ be a collection of differential forms (not necessarily closed) over $S E$ such that $\sum_{i=1}^{3 n} \operatorname{deg}\left(\eta_{i}\right)=6 n-4+\operatorname{dim} Z-1$. Then

$$
X_{n}\left(\left.E\right|_{\partial Z} ;\left(\left.\eta_{i}\right|_{S\left(\left.E\right|_{\partial Z}\right)}\right)_{i \in \underline{3 n}}\right)=\sum_{k=1}^{3 n}(-1)^{\sum_{j=1}^{k-1} \operatorname{deg}\left(\eta_{j}\right)} X_{n}\left(E ;\left(\left(\eta_{i}\right)_{i \in \underline{3 n}}\right)\left(\frac{d \eta_{k}}{\eta_{k}}\right)\right),
$$

where $\left(\left(\eta_{i}\right)_{i \in \underline{3 n}}\right)\left(d \eta_{k} / \eta_{k}\right)$ is the collection of forms obtained from $\left(\eta_{i}\right)_{i \in \underline{3 n}}$ by replacing $\eta_{k}$ with $\overline{d \eta_{k}}$.

Outline of the proof There is a natural compactification $S_{2 n}(E)$ of $\breve{S}_{2 n}(E)$; this is a compact $(6 n-4+\operatorname{dim} Z)$-dimensional manifold with corners, and there is a natural 
extension of $P_{i}(\Gamma): \breve{S}_{2 n}(E) \rightarrow S E$ to $S_{2 n}(E)$; see [8, Section 3] for details. By Stokes' theorem,

$$
\begin{aligned}
\sum_{i}^{3 n}(-1)^{\sum_{j=1}^{k-1} \operatorname{deg}\left(\eta_{j}\right)} & X_{n}\left(E ;\left(\left(\eta_{i}\right)_{i \in \underline{3 n}}\right)\left(\frac{d \eta_{k}}{\eta_{k}}\right)\right) \\
& =\sum_{\Gamma \in \mathcal{E}_{n}} \int_{S_{2 n}(E)} d\left(\bigwedge_{i} P_{i}(\Gamma)^{*} \eta_{i}\right)[\Gamma] \\
& =\sum_{\Gamma \in \mathcal{E}_{n}} \int_{\partial S_{2 n}(E)}\left(\bigwedge_{i} P_{i}(\Gamma)^{*} \eta_{i}\right)[\Gamma] \\
& =\sum_{\Gamma \in \mathcal{E}_{n}} \int_{\breve{S}_{2 n}\left(\left.E\right|_{\partial Z}\right)}\left(\left.\bigwedge_{i} P_{i}(\Gamma)^{*} \eta_{i}\right|_{S\left(\left.E\right|_{\partial Z}\right)}\right)[\Gamma] \\
& +\sum_{\Gamma \in \mathcal{E}_{n}} \int_{\partial S_{2 n}(E) \backslash \breve{S}_{2 n}\left(\left.E\right|_{\partial Z}\right)}\left(\bigwedge_{i} P_{i}(\Gamma)^{*} \eta_{i}\right)[\Gamma] .
\end{aligned}
$$

Lemmas 2,18, 2.19, 2.20 and 2.21 of [8] imply that

$$
\sum_{\Gamma \in \mathcal{E}_{n}} \int_{\partial S_{2 n}(E) \backslash \breve{S}_{2 n}\left(\left.E\right|_{\partial Z}\right)}\left(\bigwedge_{i} P_{i}(\Gamma)^{*} \eta_{i}\right)[\Gamma]=0 .
$$

The lemma is proved.

Lemma 5.2 Let $E \rightarrow Z$ and $E_{0} \rightarrow Z_{0}$ be oriented $\mathbb{R}^{3}$-bundles over manifolds with corners $Z$ and $Z_{0}$. A bundle map $f: E \rightarrow E_{0}$ induces a map $p(f): S E \rightarrow S E_{0}$. Let $\eta_{i}=p(f)^{*} \eta_{i}^{0}$ for a closed form $\eta_{i}^{0}$ on $S E_{0}$ for $i \in \underline{3 n}$ satisfying $\sum_{i=1}^{3 n} \operatorname{deg}\left(\eta_{i}\right)=$ $6 n-4+\operatorname{dim} Z$. If $\operatorname{dim} Z_{0}<\operatorname{dim} Z$, then $X_{n}\left(E ;\left(\eta_{i}\right)_{i \in 3 n}\right)=0$.

Proof We have

$$
X_{n}\left(E ;\left(\eta_{i}\right)_{i \in \underline{3 n}}\right)=\sum_{\Gamma \in \mathcal{E}_{n}} \int_{\breve{S}_{2 n}(E)}\left(\bigwedge_{i} P_{i}(\Gamma)^{*} p(f)^{*} \eta_{i}^{0}\right)[\Gamma]
$$

The map

$$
\breve{S}_{2 n}(E) \stackrel{\prod_{i} P_{i}(\Gamma)}{\longrightarrow}(S E)^{3 n} \stackrel{p(f)^{3 n}}{\longrightarrow}\left(S E_{0}\right)^{3 n}
$$

factors through $\breve{S}_{2 n}\left(E_{0}\right)$. Hence we have

$$
\left(p(f)^{3 n} \circ \prod_{i} P_{i}(\Gamma)\right)^{*} \eta_{i}^{0} \in \operatorname{Im}\left(\Omega^{6 n-4+\operatorname{dim} Z}\left(\breve{S}_{2 n}\left(E_{0}\right)\right) \rightarrow \Omega^{6 n-4+\operatorname{dim} Z}\left(\breve{S}_{2 n}(E)\right)\right) \text {. }
$$

Since

$$
\operatorname{dim} \breve{S}_{2 n}\left(E_{0}\right)=6 n-4+\operatorname{dim} Z_{0}<6 n-4+\operatorname{dim} Z,
$$


we have $\Omega^{6 n-4+\operatorname{dim} Z}\left(\breve{S}_{2 n}\left(E_{0}\right)\right)=0$. Then for any $\Gamma$, we have

$$
\int_{\breve{S}_{2 n}(E)} \bigwedge_{i} P_{i}(\Gamma)^{*} p(f)^{*} \eta_{i}^{0}=0 .
$$

\subsection{Proofs of Theorems $\mathbf{4 . 1}$ and $\mathbf{4 . 2}$}

These proofs consist of three steps.

Step 1 (well-definedness of the alternative description of the anomaly term) Here we prove the following proposition.

Proposition 5.3 There exist constants $\mu_{n} \in \mathcal{A}_{n}(\varnothing)$ such that for any closed oriented 4-manifold $X^{\prime}$ where the Euler characteristic of each component of $X^{\prime}$ is zero, for any $\mathbb{R}^{3}$-subbundle $T^{v} X^{\prime}$ of $T X^{\prime}$ and for any closed $2-$ form $W_{i}^{\prime}$ of $T^{v} X^{\prime}$ as in Section 4.1,

$$
\mu_{n} \operatorname{Sign} X^{\prime}=X_{n}\left(T^{v} X^{\prime} ;\left(W_{i}^{\prime}\right)_{i \in \underline{3 n}}\right) .
$$

To prove this proposition, we prepare a lemma.

We assume that $\operatorname{Sign} X^{\prime}=0$. Let $v_{X^{\prime}}$ be the unit vector field of $T X^{\prime}$ such that $T^{v} X^{\prime}$ is the normal bundle of $v_{X^{\prime}}$ in $T X^{\prime}$.

Lemma 5.4 There exists a compact 5-manifold $Z$ and there exists an oriented 3subbundle $T^{v} Z$ of $T Z$ such that

- $\partial Z=X^{\prime} \sqcup X^{\prime}$, and

- $\left.T^{v} Z\right|_{X^{\prime}}=T^{v} X^{\prime}$.

Proof Since $\operatorname{Sign} X^{\prime}=0$, there exists a compact connected 5-manifold $Z_{0}$ such that $\partial Z_{0}=X^{\prime}$. Let $\left.v_{Z_{0}} \in \Gamma T Z_{0}\right|_{X^{\prime}}$ be the outward unit vector field of $X^{\prime}=\partial Z_{0}$. By performing surgery along the knots generating $H_{1}\left(Z_{0} ; \mathbb{Z} / 2\right)$ if necessary, we may assume that $H_{1}\left(Z_{0} ; \mathbb{Z} / 2\right) \cong H^{4}\left(Z_{0} ; \partial Z_{0} ; \mathbb{Z} / 2\right)=0$. Thus the primary obstruction to extending the 2-framing $\left(v_{Z_{0}}, v_{X^{\prime}}\right)$ of $\left.T Z_{0}\right|_{X^{\prime}}$ into $Z_{0}$ is in $H^{5}\left(Z_{0}, \partial Z_{0} ; \pi_{4}\left(V_{5,2}\right)\right)$, where $V_{5,2}$ is the real Stiefel manifold. It is known that $\pi_{4}\left(V_{5,2}\right)=\mathbb{Z} / 2$; see $[15$, Theorem 10]. Then this primary obstruction is in $H^{5}\left(Z_{0}, \partial Z_{0} ; \mathbb{Z} / 2\right)$. Let $Z=Z_{0} \# Z_{0}$. The connected sum $Z_{0} \# Z_{0}$ is given by $\left(Z_{0} \backslash B^{5}\right) \cup[0,1] \times S^{4} \cup\left(Z_{0} \backslash B^{5}\right)$, where $B_{5}$ is a small ball in $Z_{0}$. We can extend the 2 -framing $\left(v_{Z_{0}} \sqcup v_{Z_{0}}, v_{X^{\prime}} \sqcup v_{X^{\prime}}\right)$ of $\left.T Z\right|_{X^{\prime} \sqcup X^{\prime}}$ to $Z_{0} \backslash B_{5} \sqcup Z_{0} \backslash B_{5}$. Then the obstruction to extending this 2-framing to $T Z$ is concentrated in $[0,1] \times S^{4}$. Since $H^{5}\left(S^{4} ; \pi_{4}\left(V_{5,2}\right)\right)=\mathbb{Z} / 2$, there are 
two 2-framings on $S^{4}$ up to homotopy. So the homotopy class of the 2-framing on $\{0\} \times S^{4}$ coincides with that of the 2 -framing on $\{1\} \times S^{4}$. Thus we can extend this 2 -framing to $[0,1] \times S^{4}$. Then we can also extend it to $Z$. So we can take $T^{v} Z$ as the orthonormal bundle of the $\mathbb{R}^{2}$-subbundle spanned by the extended 2 -framing.

Let $F_{Z} \rightarrow S T^{v} Z$ be the tangent bundle along the fiber of the sphere bundle $S T^{v} Z \rightarrow Z$. Let $\widetilde{W}_{i}$ be a closed 2-form on $S T^{v} Z$ that represents half of the Euler class $e\left(F_{Z}\right)$ of $F_{Z}$ and satisfies $\left.\widetilde{W}_{i}\right|_{S T^{v} X}=W_{i}$. By Lemma 5.1, we have

$$
\begin{aligned}
X_{n}\left(\left.T^{v} Z\right|_{\partial Z} ;\left(W_{i}\right)_{i \in \underline{3 n}}\right) & =\sum_{k=1}^{3 n} X_{n}\left(T^{v} Z ;\left(\left(\widetilde{W}_{i}\right)_{i \in \underline{3 n}}\right)\left(\frac{d \widetilde{W}_{k}}{\widetilde{W}_{k}}\right)\right) \\
& =0 .
\end{aligned}
$$

This lemma implies that for any closed 4-manifold $X^{\prime}$ satisfying $\chi\left(X^{\prime}\right)=0$, the number

$$
X_{n}\left(S T^{v} X^{\prime} ;\left(W_{i}^{\prime}\right)_{i \in \underline{3 n}}\right)
$$

is a cobordism invariant. In fact, for any closed 4-manifolds $X_{1}^{\prime}$ and $X_{2}^{\prime}$ satisfying $\chi\left(X_{1}^{\prime}\right)=\chi\left(X_{2}^{\prime}\right)=0$ and $\operatorname{Sign} X_{1}^{\prime}=\operatorname{Sign} X_{2}^{\prime}$ and 2 -forms $W_{1, i}^{\prime}$ and $W_{2, i}^{\prime}$ as above,

$$
\begin{aligned}
X_{n}\left(S T^{v} X_{1}^{\prime} ;\left(W_{1, i}^{\prime}\right)_{i \in \underline{3 n}}\right)-X_{n}\left(S T^{v} X_{2}^{\prime} ;\right. & \left.\left(W_{2, i}^{\prime}\right)_{i \in \underline{3 n}}\right) \\
& =X_{n}\left(S T^{v}\left(X_{1}^{\prime} \sqcup-X_{2}^{\prime}\right) ;\left(W_{1, i}^{\prime} \sqcup W_{2, i}^{\prime}\right)_{i \in \underline{3 n}}\right) \\
& =0
\end{aligned}
$$

by the above lemma.

We define a constant $\mu_{n} \in \mathcal{A}_{n}(\varnothing)$ as follows:

$$
\mu_{n}=\frac{1}{2} X_{n}\left(S T^{v}\left(\mathbb{C} P^{2} \# \mathbb{C} P^{2} \# T^{4} \# T^{4}\right) ;\left(W_{i}\right)_{i \in \underline{3 n}}\right) .
$$

Proof of Proposition 5.3 Since the cobordism group of closed 4-manifolds is $\mathbb{Z}$ and $\operatorname{Sign}\left(\mathbb{C} P^{2} \# \mathbb{C} P^{2} \# T^{4} \# T^{4}\right)=2$, we have

$$
\mu_{n} \operatorname{Sign} X^{\prime}=X_{n}\left(S T^{v} X^{\prime} ;\left(W_{i}^{\prime}\right)_{i \in \underline{3 n}}\right)
$$

for any closed 4-manifold $X^{\prime}$ with $\chi\left(X^{\prime}\right)=0$.

Let $Y$ be a closed 3-manifold with a basepoint $\infty \in Y$. Let $X$ be a compact connected 4-manifold with $\partial X=Y$ and $\chi(X)=0$. We take a collection of closed 2-forms $\left(W_{i}\right)_{i \in \underline{3 n}}$ on $S T^{v} X$ as in Section 4.1.

Corollary $5.5-\mu_{n} \operatorname{Sign} X+X_{n}\left(T^{v} X ;\left(W_{i}\right)_{i \in \underline{3 n}}\right)$ is independent of the choice of $X$ and $W_{i}$ as above. 
Proof Let $\bar{X}$ be an alternative choice for $X$. We take an $\mathbb{R}^{3}$-bundle $T^{v} \bar{X}$ and a collection of 2-forms $\left(\bar{W}_{i}\right)_{i \in \underline{3 n}}$ as above. Let $\iota:-\bar{X} \rightarrow \bar{X}$ be the identity map (but orientation reversing). Since the $\mathbb{R}^{3}$-bundles $T^{v} X$ and $\iota^{*} T^{v} \bar{X}$ are compatible on $Y$, we have an $\mathbb{R}^{3}$-subbundle $T^{v} X \cup \iota^{*} T^{v} \bar{X}$ of $T(X \cup(-\bar{X}))$. We have that $S \iota^{*} T^{v} \bar{X}$ coincides with $S T^{v} \bar{X}$ as a manifold, but it has the opposite orientation. Then the $\bar{W}_{i}$ are closed 2-forms on $S \iota^{*} T^{v} \bar{X}$ such that $\left.\bar{W}_{i}\right|_{S T(-Y)}=\left.W_{i}\right|_{S T Y}$. Therefore,

$$
\begin{aligned}
\mu_{n}(\operatorname{Sign} X-\operatorname{Sign} \bar{X}) & =X_{n}\left(T^{v} X \cup \iota^{*} T^{v} \bar{X} ;\left(W_{i} \cup \bar{W}_{i}\right)_{i \in \underline{3 n}}\right) \\
& =X_{n}\left(T^{v} X ;\left(W_{i}\right)_{i \in \underline{3 n}}\right)+X_{n}\left(\iota^{*} T^{v} \bar{X} ;\left(\bar{W}_{i}\right)_{i \in \underline{3 n}}\right) \\
& =X_{n}\left(T^{v} X ;\left(W_{i}\right)_{i \in \underline{3 n}}\right)-X_{n}\left(T^{v} \bar{X} ;\left(\bar{W}_{i}\right)_{i \in \underline{\underline{n}}}\right) .
\end{aligned}
$$

Step $2\left(z_{n}\left(\left(\omega_{i}\right)_{i \in \underline{3 n}}\right)+\mu_{n} \operatorname{Sign} X-X_{n}\left(T^{v} X ;\left(W_{i}\right)_{i \in \underline{3 n}}\right)\right.$ is a topological invariant of $\left.Y\right)$ Let $Y$ be a rational homology 3 -sphere and $X$ a compact connected 4-manifold with $\partial X=Y$ and $\chi(X)=0$. Let $\tau_{Y}$ be an admissible framing of $T(Y \backslash \infty)$. Let $\tau_{Y}^{\prime}=\left.\left.\tau_{Y}\right|_{B_{Y}} \cup \tau_{S^{3}}\right|_{N\left(\infty ; S^{3}\right)}$. So $\tau_{Y}^{\prime}$ is a framing of $T Y$. Take a $2-$ form $W\left(\tau_{Y}, \omega_{S^{2}, i}\right)$ on $X$ as in Section 4.1 such that $\left.W\left(\tau_{Y}, \omega_{S^{2}, i}\right)\right|_{S T Y}=p\left(\tau_{Y}^{\prime}\right)^{*} \omega_{S^{2}, i}$ for a volume form $\omega_{S^{2}, i}$ on $S^{2}$.

Lemma 5.6 We have that $X_{n}\left(T^{v} X ;\left(W\left(\tau_{Y}, \omega_{S^{2}, i}\right)\right)_{i \in \underline{3 n}}\right)$ is independent of the choice of $\omega_{S^{2}, 1}, \ldots, \omega_{S^{2}, 3 n}$.

Proof Let $\omega_{S^{2}, i}^{\prime}$ be an alternative choice for $\omega_{S^{2}, i}$. Since we have $\left[\omega_{S^{2}, i}^{\prime}\right]=$ $\left[\omega_{S^{2}, i}\right] \in H^{2}\left(S^{2} ; \mathbb{R}\right)$, there exists a closed 2 -form $\Omega_{S^{2}, i}$ on $[0,1] \times S^{2}$ that satisfies $\left.\Omega_{S^{2}, i}\right|_{\{0\} \times S^{2}}=\omega_{S^{2}, i}$ and $\left.\Omega_{S^{2}, i}\right|_{\{1\} \times S^{2}}=\omega_{S^{2}, i}^{\prime}$.

Let $[0,1] \times S T Y \subset S T^{v} X$ be the collar of $S T^{v} X$ such that $\{0\} \times S T Y=\partial S T^{v} X$. We take $\left.W\left(\tau_{Y}, \omega_{S^{2}, i}\right)\right|_{[0,1] \times S T Y}=\left(\operatorname{id} \times p\left(\tau_{Y}\right)\right)^{*} \Omega_{S^{2}, i}$. Thus $\left.W\left(\tau_{Y}, \omega_{S^{2}, i}\right)\right|_{\{1\} \times S T Y}=$ $p\left(\tau_{Y}^{\prime}\right)^{*} \omega_{S^{2}, i}^{\prime}$. Then we have

$$
\begin{aligned}
X_{n}\left(T^{v} X ;\left(W\left(\tau_{Y}, \omega_{S^{2}, i}\right)\right)_{i \in \underline{3 n}}\right)-X_{n}\left(T^{v} X ;\left(W\left(\tau_{Y}, \omega_{S^{2}, i}^{\prime}\right)\right)_{i \in \underline{3 n}}\right) \\
=\sum_{\Gamma \in \mathcal{E}_{n}} \int_{[0,1] \times \breve{S}_{2 n}(T Y)} \bigwedge_{i} P_{i}(\Gamma)^{*} W\left(\tau_{Y}, \omega_{S^{2}, i}\right)[\Gamma] \\
=\sum_{\Gamma \in \mathcal{E}_{n}} \int_{[0,1] \times \breve{S}_{2 n}(T Y)} \bigwedge_{i} P_{i}(\Gamma)^{*}\left(\mathrm{id} \times p\left(\tau_{Y}\right)\right)^{*} \Omega_{S^{2}, i}[\Gamma] .
\end{aligned}
$$

In Lemma 5.2, we take

$$
\begin{aligned}
& Z=[0,1] \times Y, \quad E=[0,1] \times T Y, \quad f=\mathrm{id} \times\left(p \circ \tau_{Y}\right), \\
& Z_{0}=[0,1] \times\{\mathrm{pt}\}, \quad E_{0}=[0,1] \times \mathbb{R}^{3}, \quad \eta_{i}=\Omega_{S^{2}, i} .
\end{aligned}
$$


Here $p: \underline{\mathbb{R}^{3}} \rightarrow \mathbb{R}^{3}$ is the standard projection. For any $\Gamma \in \mathcal{E}_{n}$, we have

$$
\int_{[0,1] \times \breve{S}_{2 n}(T Y)} \bigwedge_{i} P_{i}(\Gamma)^{*}\left(\mathrm{id} \times p\left(\tau_{Y}\right)\right)^{*} \Omega_{S^{2}, i}=0 .
$$

This completes the proof.

Thanks to Corollary 5.5 and Lemma 5.6,

$$
-\mu_{n} \operatorname{Sign} X_{S^{3}}+X_{n}\left(T^{v} S^{3} ;\left(W\left(\tau_{\mathbb{R}^{3}}, \omega_{S^{2}, i}\right)\right)_{i \in \underline{3 n}}\right)
$$

is independent of the choice of a 4-manifold $X_{S^{3}}$ bounded by $S^{3}$ and of the forms $\omega_{S^{2}, 1}, \ldots, \omega_{S^{2}, 3 n}$. We define

$$
c_{n}=-\mu_{n} \operatorname{Sign} X_{S^{3}}+X_{n}\left(T^{v} S^{3} ;\left(W\left(\tau_{\mathbb{R}^{3}}, \omega_{S^{2}, i}\right)\right)_{i \in \underline{3 n}}\right) \in \mathcal{A}_{n}(\varnothing) .
$$

Let $I_{n}\left(\left(\omega_{i}\right)_{i \in \underline{3 n}}\right)=-\mu_{n} \operatorname{Sign} X+X_{n}\left(T^{v} X ;\left(W_{i}\right)_{i \in \underline{3 n}}\right)-c_{n}$.

Proposition $5.7 z_{n}\left(\left(\omega_{i}\right)_{i \in \underline{3 n}}\right)-I_{n}\left(\left(\omega_{i}\right)_{i \in \underline{3 n}}\right)$ is a topological invariant of $Y$.

Proof Let $\omega_{S^{2}, i}^{\prime}$ and $\omega_{i}^{\prime}$ be alternative choices for $\omega_{S^{2}, i}$ and $\omega_{i}$, respectively. We take a corresponding 2-form $W_{i}^{\prime}$ in $S T^{v} X$.

By Lemma 3.5, there exist closed 2-forms $\Omega_{i}$ on $[0,1] \times S T B_{Y}$ such that

$$
z_{n}\left(\left(\omega_{i}^{\prime}\right)_{i \in \underline{3 n}}\right)-z_{n}\left(\left(\omega_{i}\right)_{i \in \underline{3 n}}\right)=X_{n}\left([0,1] \times T B_{Y} ;\left(\Omega_{i}\right)_{i \in \underline{3 n}}\right) .
$$

Let $\tilde{X}=X \cup_{\partial X=\{0\} \times Y}[0,1] \times Y \cup_{\{1\} \times Y=\partial X}-X$. Then

$$
\widetilde{\Omega}_{i}=W_{i} \cup\left(\Omega_{i} \cup\left(\operatorname{id} \times\left. p\left(\tau_{S^{3}}\right)\right|_{N(\infty ; Y)}\right)^{*} \Omega_{S^{2}, i}\right) \cup W_{i}^{\prime}
$$

is a closed 2-form on $S T^{v} \tilde{X}=S T^{v} X \cup[0,1] \times S T Y \cup-S T^{v} X$. Thanks to Proposition 5.3, $X_{n}\left(T^{v} \tilde{X} ;\left(\tilde{\Omega}_{i}\right)_{i \in \underline{3 n}}\right)=\mu_{n} \operatorname{Sign} \tilde{X}=0$. Thus

$I_{n}\left(\left(\omega_{i}^{\prime}\right)_{i \in \underline{3 n}}\right)-I_{n}\left(\left(\omega_{i}\right)_{i \in \underline{3 n}}\right)$

$$
\begin{aligned}
= & X_{n}\left(T^{v} X ;\left(W_{i}^{\prime}\right)_{i \in \underline{3 n}}\right)-X_{n}\left(T^{v} X ;\left(W_{i}\right)_{i \in \underline{3 n}}\right) \\
=- & X_{n}\left(T^{v} \tilde{X} ;\left(\widetilde{\Omega}_{i}\right)_{i \in \underline{3 n}}\right) \\
& \quad+X_{n}\left([0,1] \times T Y ;\left(\Omega_{i} \cup\left(\left(\mathrm{id} \times\left. p\left(\tau_{S^{3}}\right)\right|_{N(\infty ; Y)}\right)^{*} \Omega_{S^{2}, i}\right)_{i \in \underline{3 n}}\right)\right. \\
= & X_{n}\left([0,1] \times T Y ;\left(\Omega_{i} \cup\left(\mathrm{id} \times\left. p\left(\tau_{S^{3}}\right)\right|_{N(\infty ; Y)}\right)^{*} \Omega_{S^{2}, i}\right)_{i \in \underline{3 n}}\right) \\
= & X_{n}\left([0,1] \times T B_{Y} ;\left(\Omega_{i}\right)_{i \in \underline{3 n}}\right) \\
& \quad+X_{n}\left([0,1] \times T N(\infty ; Y) ;\left(\left(\mathrm{id} \times\left. p\left(\tau_{S^{3}}\right)\right|_{N(\infty ; Y)}\right)^{*} \Omega_{S^{2}, i}\right)_{i \in \underline{3 n}}\right) .
\end{aligned}
$$


Thanks to Lemma 5.2, $X_{n}\left([0,1] \times T N(\infty ; Y) ;\left(\left(\mathrm{id} \times p\left(\tau_{S^{3}}\right)\right)^{*} \Omega_{S^{2}, i}\right)_{i \in \underline{3 n}}\right)=0$. Thus $I_{n}\left(\left(\omega_{i}^{\prime}\right)_{i \in \underline{3 n}}\right)-I_{n}\left(\left(\omega_{i}\right)_{i \in \underline{3 n}}\right)=X_{n}\left([0,1] \times T B_{Y} ;\left(\Omega_{i}\right)_{i \in \underline{3 n}}\right)$. Therefore,

$$
z_{n}\left(\left(\omega_{i}\right)_{i \in \underline{3 n}}\right)-I_{n}\left(\left(\omega_{i}\right)_{i \in \underline{3 n}}\right)-\left(z_{n}\left(\left(\omega_{i}^{\prime}\right)_{i \in \underline{3 n}}\right)-I_{n}\left(\left(\omega_{i}^{\prime}\right)_{i \in \underline{3 n}}\right)\right)=0 .
$$

Step 3 (proof of $I_{n}\left(\left(\omega_{i}\right)_{i \in \underline{3 n}}\right)=\widetilde{z}_{n}^{\text {anomaly }}\left(\left(\omega_{i}\right)_{i \in \underline{3 n}}\right)$ ) Recall that $\widetilde{z}_{n}^{\text {anomaly }}\left(\left(\omega_{i}\right)_{i \in \underline{3 n}}\right)$ is defined in Definition 3.9. We next prove $I_{n}\left(\left(\omega_{i}\right)_{i \in \underline{3 n}}\right)=\widetilde{z}_{n}^{\text {anomaly }}\left(\left(\omega_{i}\right)_{i \in \underline{3 n}}\right)$ and determine constants $\mu_{n}$ and $c_{n}$.

Let $Y$ be a closed 3-manifold (which may not be a rational homology 3-sphere) with a basepoint $\infty$. We denote $I_{n}\left(\tau_{Y}\right)=I_{n}\left(\left(\omega_{\tau_{Y}}\right)_{i \in \underline{3 n}}\right)$ for an admissible framing $\tau_{Y}$ of $T(Y \backslash \infty)$.

Since Definition 3.9 and Proposition 5.7 imply that $I_{n}\left(\left(\omega_{i}\right)_{i \in \underline{3 n}}\right)-\widetilde{z}_{n}^{\text {anomaly }}\left(\left(\omega_{i}\right)_{i \in \underline{3 n}}\right)$ does not depend on the $\omega_{i}$, we only need to show that

$$
I_{n}\left(\tau_{Y}\right)=-\frac{1}{4} \sigma_{Y \backslash \infty}\left(\tau_{Y}\right) \delta_{n}
$$

for some admissible framing $\tau_{Y}$ of $T(Y \backslash \infty)$.

\section{Lemma 5.8}

$$
I_{n}\left(\tau_{\mathbb{R}^{3}}\right)=-\frac{1}{4} \sigma_{\mathbb{R}^{3}}\left(\tau_{\mathbb{R}^{3}}\right) \delta_{n}=0 .
$$

Proof Because the definition of $c_{n}$, we have $I_{n}\left(\left(\omega_{\tau_{\mathbb{R}^{3}}}\right)_{i \in \underline{3 n}}\right)=0$.

Since $z_{n}^{\mathrm{KKT}}\left(S^{3} ; \tau\right)-I_{n}\left(\left(\omega_{\tau}\right)_{i \in \underline{3 n}}\right)$ is independent of the choice of an admissible framing $\tau$ of $T \mathbb{R}^{3}$, we have the following corollary.

Corollary 5.9 For any admissible framing $\tau$ of $T \mathbb{R}^{3}$, the equation $I_{n}\left(\left(\omega_{\tau}\right)_{i \in \underline{3 n}}\right)=$ $-\frac{1}{4} \sigma_{\mathbb{R}^{3}}(\tau) \delta_{n}$ holds.

We introduce a cobordism group $\Omega_{3}^{\text {Sign=0 }}$, generated by all framed closed 3-manifolds $(Y, \tau)$ and divided by a cobordism relation $\sim:(Y, \tau) \sim \varnothing$ if and only if there exists a pair $\left(X, \tau_{X}\right)$ such that

- $\quad$ Sign $X=0$, and

- $\tau_{X}$ is a 3-framing of $T X$ such that $\left.\tau_{X}\right|_{Y}=\tau$.

For a framed 3-manifold $(Y, \tau)$, set $J_{n}(\tau)=-\mu_{n} \operatorname{Sign} X+X_{n}\left(T^{v} X ;\left(W_{\tau}\right)_{i \in \underline{3 n}}\right)$. Here $X$ and $T^{v} X$ are same as above, and $W_{\tau}$ is a closed 2-form of $S T^{v} X$ satisfying $\left[W_{\tau}\right]=\frac{1}{2} e\left(F_{X}\right)$ and $\left.W_{\tau}\right|_{S T Y}=p(\tau)^{*} \omega_{S^{2}}$. Then $I_{n}\left(\tau_{Y}\right)=J_{n}\left(\tau_{Y}^{\prime}\right)-c_{n}$. Recall that $\sigma_{Y \backslash \infty}\left(\tau_{Y}\right)=\sigma_{Y}\left(\tau_{Y}^{\prime}\right)-2$.

Lemma 5.10 Both $J_{n}(\bullet)$ and $-\frac{1}{4} \sigma_{\bullet}(\bullet) \delta_{n}$ factor through $\Omega_{3}^{\text {Sign }=0}$. 
Proof By definition of the signature defect, $\sigma_{\bullet}(\bullet)$ is obviously a cobordism invariant.

We show that $J_{n}(\bullet)$ is a cobordism invariant. Let $\left(X, \tau_{X}\right)$ be a cobordism between a framed 3-manifold $\left(Y,\left.\tau_{X}\right|_{Y}\right)$ and $\varnothing$. Let $T^{v} X$ be the $\mathbb{R}^{3}$-subbundle of $T X$ spanned by $\tau_{X}$. Then $\tau_{X}$ induces the map $p\left(\tau_{X}\right): S T^{v} X \rightarrow S^{2}$. Take $W_{\tau_{X}}=p\left(\tau_{X}\right)^{*} \omega_{S^{2}}$. Then we have

$$
\begin{aligned}
J_{n}\left(\left.\tau_{X}\right|_{Y}\right) & =-\mu_{n} \operatorname{Sign} X+X_{n}\left(T^{v} X ;\left(W_{\tau_{X}}\right)_{i \in \underline{3 n}}\right) \\
& =\sum_{\Gamma \in \mathcal{E}_{n}} \int_{\breve{S}_{2 n}\left(T^{v} X\right)} \bigwedge_{i} P_{i}(\Gamma)^{*} p\left(\tau_{X}\right)^{*} \omega_{S^{2}}[\Gamma]
\end{aligned}
$$

Lemma 5.2 implies that the above integral vanishes for any $\Gamma \in \mathcal{E}_{n}$. Therefore, $J_{n}\left(\left.\tau_{X}\right|_{Y}\right)=0$.

Lemma 5.11 (1) $\Omega_{3}^{\text {Sign }=0} \otimes_{\mathbb{Z}} \mathbb{Q}=\mathbb{Q}$ and $\left(S^{3}, \tau_{S^{3}}\right)$ is a generator.

(2) $c_{n}=-\frac{1}{2} \delta_{n}$.

(3) For any framed closed 3-manifold $(Y, \tau), J_{n}(\tau)=-\frac{1}{4} \sigma_{Y}(\tau) \delta_{n}$.

(4) $\mu_{n}=-\frac{3}{4} \delta_{n}$.

Proof (1) Let $(Y, \tau)$ be a framed closed 3-manifold. We use the same symbol $(Y, \tau)$ for its cobordism class. We give the spin structure on $Y$ using $\tau$. Since the 3 -dimensional spin cobordism group is equal to zero, there exists a spin 4-manifold $X$ such that $\partial X=Y$. Let $k=\chi(X)$. We may assume that $k \geq 0$, by replacing $X$ by $X \# n\left(S^{2} \times S^{2}\right)$ for a sufficiently large integer $n$ if necessary. Let $X_{0}$ be the spin 4manifold obtained by removing $k$ disjoint 4-balls; ie $X_{0}=X \backslash k B^{4}$. Then $\chi\left(X_{0}\right)=0$ and $\partial X_{0}=Y \sqcup k\left(-S^{3}\right)$. Here $-S^{3}$ is $S^{3}$ with the opposite orientation. We denote by $-S_{i}^{3}$ the $i^{\text {th }}\left(-S^{3}\right)$-boundary of $X_{0}$. Then $\partial X_{0}=Y \sqcup-S_{1}^{3} \sqcup \cdots \sqcup-S_{k}^{3}$.

By obstruction theory, there is a 3 -framing $\tau_{X_{0}}$ of $T X_{0}$ that satisfies $\left.\tau_{X_{0}}\right|_{Y}=\tau$, and $\tau_{X_{0}} \mid-S_{i}^{3}$ is a framing of $T\left(-S_{i}^{3}\right)$ for each $i$; see [5] for more details. Let $\tau_{i}=$ $-\left(\tau_{X_{0}} \mid-S_{i}^{3}\right)$. Thus $(Y, \tau)=\sum_{i=1}^{k}\left(S^{3}, \tau_{i}\right)$ in $\Omega_{3}^{\text {Sign }=0}$.

Let $\tau^{s}$ be any framing of $T S^{3}$. It is known that $\sigma_{S^{3}}\left(\tau^{s}\right) \in 4 \mathbb{Z}+2$; see [5]. Let $X_{1}=\left(S^{4} \backslash\left(\left|\sigma_{S^{3}}\left(\tau^{s}\right)\right|+2\right) B^{4}\right) \# \frac{1}{2}\left|\sigma_{S^{3}}\left(\tau^{s}\right)\right|\left(S^{2} \times S^{2}\right)$. Then $X_{1}$ is a spin 4-manifold satisfying $\operatorname{Sign}\left(X_{1}\right)=0, \chi\left(X_{1}\right)=0$ and $\partial X_{1}=\left(S^{3} \sqcup S^{3}\right) \sqcup\left|\sigma\left(\tau^{s}\right)\right|\left(-S^{3}\right)$. By obstruction theory, there exists a 3-framing $\tau_{X_{1}}$ of $T X$ such that $\left.\tau_{X_{1}}\right|_{S^{3}}=\tau^{s}$ and $\left.\tau_{X_{1}}\right|_{-S^{3}}=\tau_{S^{3}}^{+}$or $\left.\tau_{X_{1}}\right|_{-S^{3}}=\tau_{S^{3}}^{-}$, where $\tau_{S^{3}}^{ \pm}$is a framing of $T S^{3}$ that satisfies $\sigma_{S^{3}}\left(\tau_{S^{3}}^{ \pm}\right)= \pm 2$, respectively. Thus $2(Y, \tau)=n_{+}\left(S^{3}, \tau_{S^{3}}^{+}\right)+n_{-}\left(S^{3}, \tau_{S^{3}}^{-}\right)$in $\Omega_{3}^{\text {Sign }=0}$ for some nonnegative integers $n_{+}$and $n_{-}$.

The spin 4-manifold $[0,1] \times S^{3}$ is a cobordism between $\left(S^{3}, \tau_{S^{3}}^{+}\right) \sqcup\left(S^{3}, \tau_{S^{3}}^{-}\right)$and $\varnothing$. Therefore, we have $2(Y, \tau)=\left(n_{+}-n_{-}\right)\left(S^{3}, \tau_{S^{3}}^{+}\right)$in $\Omega_{3}^{\text {Sign }=0}$. 
(2) Since $0=I_{n}\left(\tau_{\mathbb{R}^{3}}\right)=J_{n}\left(\tau_{S^{3}}\right)-c_{n}$, we have $J_{n}\left(\tau_{S^{3}}\right)=c_{n}$. Let $\tau_{S^{3}}^{-}$be a framing of $T S^{3}$ satisfying $\sigma_{S^{3}}\left(\tau_{S^{3}}^{-}\right)=-2$. Since $J_{n}(\bullet)$ is a cobordism invariant, $J_{n}\left(\tau_{S^{3}}^{-}\right)=-J_{n}\left(\tau_{S^{3}}\right)=-c_{n}$. Thanks to Corollary 5.9,

$$
-2 c_{n}=J_{n}\left(\tau_{S^{3}}^{-}\right)-c_{n}=-\frac{1}{4} \sigma_{S^{3}}\left(\tau_{S^{3}}^{-}\right) \delta_{n}+\frac{1}{2} \delta_{n}=\delta_{n} .
$$

Then we have $c_{n}=-\frac{1}{2} \delta_{n}$.

(3) $J_{n}\left(\tau_{S^{3}}\right)=c_{n}=-\frac{1}{2} \delta_{n}=-\frac{1}{4} \sigma_{S^{3}}\left(\tau_{S^{3}}\right) \delta_{n}$ and $\tau_{S^{3}}$ is a generator.

(4) Recall that $-K 3$ is a spin 4-manifold with $\chi(-K 3)=24$ and $\operatorname{Sign}(-K 3)=16$. Let $X_{a}=\left(-K_{3}\right) \# 11 T^{4} \backslash\left(B^{4} \sqcup B^{4}\right)$. Then $X_{a}$ is a spin 4-manifold satisfying $\chi\left(X_{a}\right)=0$ and $\operatorname{Sign}\left(X_{a}\right)=16$. It is possible to deal with $\partial X_{a}=S^{3} \sqcup-S^{3}$. There is a 3 -framing $\tau_{X_{a}}$ of $T X_{a}$ such that $\left.\tau_{X_{a}}\right|_{\partial X_{a}}$ is a framing of $T\left(\partial X_{a}\right)$. The 3-framing $\tau_{X_{a}}$ spans the $\mathbb{R}^{3}$-subbundle $T^{v} X_{a}$ of $T X_{a}$. Let $W=p\left(\tau_{X_{a}}\right)^{*} \omega_{S^{2}}$ be the closed 2-form on $S T^{v} X_{a}$. Thanks to Lemma 5.2, $X_{n}\left(T^{v} X_{a} ;(W)_{i \in 3 n}\right)=0$. Then we have

$$
\begin{aligned}
J_{n}\left(\left.\tau_{X_{a}}\right|_{\partial X_{a}}\right) & =-\mu_{n} \operatorname{Sign} X_{a}+X_{n}\left(T^{v} X_{a} ;(W)_{i \in \underline{3 n}}\right) \\
& =-16 \mu_{n} .
\end{aligned}
$$

By (3) and the definition of the signature defect, we have

$$
J_{n}\left(\left.\tau_{X_{a}}\right|_{\partial X_{a}}\right)=-\frac{1}{4} \sigma_{\partial X_{a}}\left(\left.\tau_{X_{a}}\right|_{\partial X_{a}}\right) \delta_{n}=\frac{3}{4} \operatorname{Sign} X_{a} \delta_{n}=12 \delta_{n} .
$$

Therefore $\mu_{n}=-\frac{3}{4} \delta_{n}$.

Corollary 5.12 For any admissible framing $\tau_{Y}$ of $T(Y \backslash \infty)$,

$$
I_{n}\left(\tau_{Y}\right)=-\frac{1}{4} \sigma_{Y \backslash \infty}\left(\tau_{Y}\right) \delta_{n} .
$$

Proof $I_{n}\left(\tau_{Y}\right)=J_{n}\left(\tau_{Y}^{\prime}\right)-c_{n}=-\frac{1}{4} \sigma_{Y}\left(\tau_{Y}^{\prime}\right) \delta_{n}+\frac{1}{2} \delta_{n}=-\frac{1}{4} \sigma_{Y \backslash \infty}\left(\tau_{Y}\right) \delta_{n}$.

\subsection{Proof of Theorem 4.7}

Let $f$ be an admissible Morse function with respect to $a \in S^{2}$. Let $\operatorname{Crit}(f)=$ $\left\{p_{1}, \ldots, p_{k}, q_{1}, \ldots, q_{k}\right\}$ where $\operatorname{ind}\left(p_{i}\right)=2$ and $\operatorname{ind}\left(q_{i}\right)=1$. Let

$$
\widehat{\partial}: C_{2}(Y \backslash \infty ; f) \rightarrow C_{1}(Y \backslash \infty ; f), \quad \widehat{\partial}\left[p_{i}\right]=\sum_{j} \widehat{\partial}_{i j}\left[q_{j}\right],
$$

be the boundary map of the Morse complex.

We first give a compactification $\mathcal{M}_{S}( \pm f)$ of $\mathcal{M}(f) \cup \mathcal{M}(-f)$ and then show that $\mathcal{M}_{S}( \pm f)$ is a 4-cycle in $\left(C_{2}(Y), \partial C_{2}(Y)\right)$. Recall that the 4-manifold $M_{\rightarrow}(f)$ is the image of the inclusion map

$$
\varphi_{f}:(Y \backslash \infty) \times(0, \infty) \hookrightarrow(Y \backslash \infty) \times(Y \backslash \infty), \quad(x, t) \mapsto\left(x, \Phi_{f}^{t}(x)\right),
$$


and the map $\varphi_{f}$ preserves orientations. Let $\bar{M}_{\rightarrow}(f)$ be the closure of $M_{\rightarrow}(f)$ in $(Y \backslash \infty)^{2} \backslash \Delta$. With our orientation conventions, the following two lemmas hold.

Lemma 5.13 [11, Lemma 4.3] [14, Proposition 3.4] $\bar{M}_{\rightarrow}(f)$ is a manifold with corners satisfying the following conditions:

(1) int $\bar{M}_{\rightarrow}(f)=M_{\rightarrow}(f)$, and

(2) $\partial \bar{M}_{\rightarrow}(f)=-\sum_{i} \mathcal{D}_{p_{i}} \times \mathcal{A}_{p_{i}}-\sum_{j} \mathcal{D}_{q_{j}} \times \mathcal{A}_{q_{j}}$.

Lemma 5.14 [14, Proposition 3.17] There are manifolds with corners $\overline{\mathcal{A}}_{q_{j}}$ and $\overline{\mathcal{D}}_{p_{i}}$ satisfying the following conditions:

(1) int $\overline{\mathcal{A}}_{q_{j}}=\mathcal{A}_{q_{j}}$, int $\overline{\mathcal{D}}_{p_{i}}=\mathcal{D}_{p_{i}}$,

(2) $\partial \overline{\mathcal{A}}_{q_{j}}=-\sum_{i} \hat{\partial}_{i j} \mathcal{A}_{p_{i}}, \partial \overline{\mathcal{D}}_{p_{i}}=-\sum_{j} \widehat{\partial}_{i j} \mathcal{D}_{q_{j}}$.

We denote by $\overline{\mathcal{A}}_{p_{i}} \rightarrow Y$ the extension of $\mathcal{A}_{p_{i}} \rightarrow Y$ to $\overline{\mathcal{A}}_{p_{i}}$ and we write $\overline{\mathcal{A}}_{p_{i}}$ instead of $\overline{\mathcal{A}}_{p_{i}} \rightarrow Y$. We also define $\overline{\mathcal{D}}_{p_{i}}, \overline{\mathcal{A}}_{q_{j}}$, and so on.

Definition 5.15 (1) $\mathcal{M}_{S}(f)$ is the closure, in $C_{2}(Y)$, of

$$
\bar{M}_{\rightarrow}(f)+\sum_{i, j} \hat{g}_{j i}\left(\left(\overline{\mathcal{D}}_{p_{i}} \times \overline{\mathcal{A}}_{q_{j}}\right) \cap(Y \backslash \infty)^{2} \backslash \Delta\right) .
$$

(2) $\mathcal{M}_{S}( \pm f)=\mathcal{M}_{S}(f)+\mathcal{M}_{S}(-f)$.

Thanks to the Morse-Smale condition, $M_{S}(f)$ and $M_{S}( \pm f)$ are 4-chains of $C_{2}(Y)$.

Lemma 5.16 $\mathcal{M}_{S}(f), \mathcal{M}_{S}(-f)$ and $\mathcal{M}_{S}( \pm f)$ are 4-cycles in $\left(C_{2}(Y), \partial C_{2}(Y)\right)$.

Proof Since $\operatorname{Im}\left(\partial\left(\overline{\mathcal{D}}_{p_{i}} \times \overline{\mathcal{A}}_{q_{j}}\right) \rightarrow Y^{2}\right)=-\sum_{k} \widehat{\partial}_{k j} \overline{\mathcal{D}}_{p_{i}} \times \overline{\mathcal{A}}_{p_{k}}-\sum_{k} \widehat{\partial}_{i k} \overline{\mathcal{D}}_{q_{k}} \times \overline{\mathcal{A}}_{q_{j}}$,

$$
\begin{aligned}
\operatorname{Im}\left(\sum_{i, j} \hat{g}_{j i} \partial\left(\overline{\mathcal{D}}_{p_{i}} \times \overline{\mathcal{A}}_{q_{j}} \rightarrow Y^{2}\right)\right) & =-\sum_{i, j, k} \hat{g}_{j i} \hat{\partial}_{k j} \overline{\mathcal{D}}_{p_{i}} \times \overline{\mathcal{A}}_{p_{k}}-\sum_{i, j, k} \hat{g}_{j i} \hat{\partial}_{i k} \overline{\mathcal{D}}_{q_{k}} \times \overline{\mathcal{A}}_{q_{j}} \\
& =-\sum_{i, j, k} \delta_{k i} \overline{\mathcal{D}}_{p_{i}} \times \overline{\mathcal{A}}_{p_{k}}-\sum_{i, j, k} \delta_{j k} \overline{\mathcal{D}}_{q_{k}} \times \overline{\mathcal{A}}_{q_{j}} \\
& =-\sum_{i} \overline{\mathcal{D}}_{p_{i}} \times \overline{\mathcal{A}}_{p_{i}}-\sum_{j} \overline{\mathcal{D}}_{q_{j}} \times \overline{\mathcal{A}}_{q_{j}} \\
& =-\partial \bar{M}_{\rightarrow}(f) .
\end{aligned}
$$

Therefore, $\partial \mathcal{M}_{S}(f) \backslash \partial C_{2}(Y)=\varnothing$. 


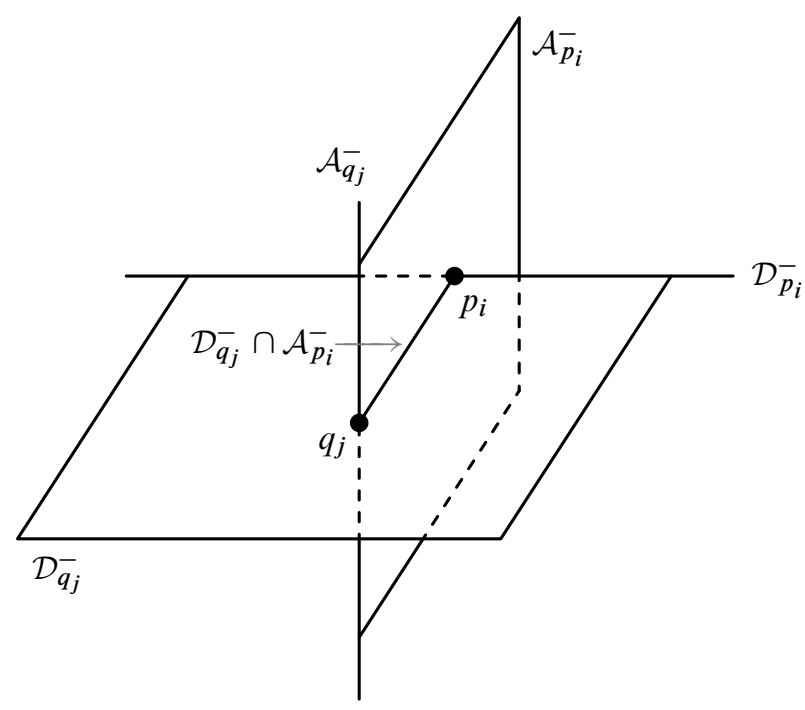

Figure 3: The orientation of $\mathcal{D}_{q_{j}}^{-} \cap \mathcal{A}_{p_{i}}^{-}$

The critical points of the Morse function $(-f: Y \backslash \infty \rightarrow \mathbb{R})$ form the set Crit $(-f)=$ $\left\{q_{1}, \ldots, q_{k}, p_{1}, \ldots, p_{k}\right\}$, where $\operatorname{ind}\left(q_{i}\right)=2$ and $\operatorname{ind}\left(p_{i}\right)=1$. We remark that the ascending manifold $\mathcal{A}_{p_{i}}^{-}$of $p_{i}$ corresponding to $-f$ is $\mathcal{D}_{p_{i}}$, and the descending manifold $\mathcal{D}_{q_{j}}^{-}$of $q_{j}$ corresponding to $-f$ is $\mathcal{A}_{q_{j}}$. We orient $\mathcal{A}_{p_{i}}^{-}$as $\mathcal{D}_{p_{i}}$ and orient $\mathcal{D}_{q_{j}}^{-}$ as $\mathcal{A}_{q_{j}}$.

Lemma 5.17 With our conventions, the orientations of $\mathcal{D}_{q_{j}}^{-} \cap \mathcal{A}_{p_{i}}^{-}$and $\mathcal{D}_{p_{i}} \cap \mathcal{A}_{q_{j}}$ are opposite.

Proof Let $n\left(\mathcal{D}_{p_{i}}\right)$ and $n\left(\mathcal{A}_{q_{j}}\right)$ be positively oriented normal vector fields of $\mathcal{D}_{p_{i}}$ and $\mathcal{A}_{q_{j}}$, respectively. Then $n\left(\mathcal{D}_{p_{i}}\right)$ and $n\left(\mathcal{A}_{q_{j}}\right)$ are positively oriented normal vector fields of $\mathcal{A}_{p_{i}}^{-}$and $\mathcal{D}_{q_{j}}^{-}$, respectively. Since the orientation of the normal bundle $N\left(\mathcal{D}_{p_{i}} \cap \mathcal{A}_{q_{j}}\right)$ induced by the frame $\left(\left.\left.n\left(\mathcal{A}_{q_{j}}\right)\right|_{\mathcal{D}_{p_{i}} \cap \mathcal{A}_{q_{j}}} n\left(\mathcal{D}_{p_{i}}\right)\right|_{\mathcal{D}_{p_{i}} \cap \mathcal{A}_{q_{j}}}\right)$ is opposite to

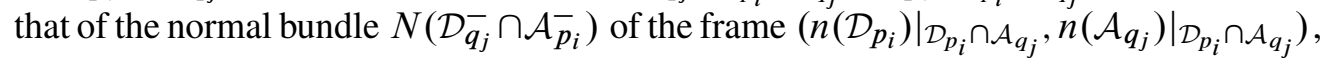
the orientation of $\mathcal{D}_{q_{j}}^{-} \cap \mathcal{A}_{p_{i}}^{-}$is opposite to that of $\mathcal{D}_{p_{i}} \cap \mathcal{A}_{q_{j}}$. (See Figure 3.)

By this lemma, the sign of each component of $\mathcal{D}_{q_{j}}^{-} \cap \mathcal{A}_{p_{i}}^{-}$coincides with the sign of the corresponding component in $\mathcal{D}_{p_{i}} \cap \mathcal{A}_{q_{j}}$. So the boundary map $\hat{\partial}_{\bar{j} i}$ (resp. $\hat{g}_{i j}^{-}$) corresponding to $-f$ is equal to $\hat{\partial}_{i j}$ (resp. $\hat{g}_{j i}$ ) corresponding to $f$. Thus $\mathcal{M}_{S}(-f)$ is equal to the closure of $\bar{M}_{\rightarrow}(-f)+\sum_{i, j} \hat{g}_{j i}\left(\left(\overline{\mathcal{A}}_{q_{j}} \times \overline{\mathcal{D}}_{p_{i}}\right) \cap(Y \backslash \infty)^{2} \backslash \Delta\right)$ with our orientation conventions. 
Furthermore, we have the following description.

Lemma 5.18 $\left.\partial \mathcal{M}_{S}( \pm f) \cap S T B_{Y}=\overline{\left\{\operatorname{grad}_{x} f\right],\left[-\operatorname{grad}_{x} f\right] \mid x \in B_{Y} \backslash \operatorname{Crit}(f)}\right\}$.

Proof Note that $\left(\overline{\mathcal{D}}_{p_{i}} \times \overline{\mathcal{A}}_{q_{j}}\right) \cap \Delta=\overline{\mathcal{D}_{p_{i}} \cap \mathcal{A}_{q_{j}}}$. There exists $\varepsilon \in\{-1,1\}$ such that the oriented boundary of the closure of $\left(\mathcal{D}_{p_{i}} \times \mathcal{A}_{q_{j}}\right) \cap\left((Y \backslash \infty)^{2} \backslash \Delta\right)$ meets $S T B_{Y}$ as $\varepsilon \pi^{-1}\left(\overline{\mathcal{D}_{p_{i}} \cap \mathcal{A}_{q_{j}}}\right)$, where $\pi^{-1}\left(\mathcal{D}_{p_{i}} \cap \mathcal{A}_{q_{j}}\right)$ is oriented as the product of $\mathcal{D}_{p_{i}} \cap \mathcal{A}_{q_{j}}$ by the fiber of $S T B_{Y}$, which has a canonical orientation. Here $\pi: S T Y \rightarrow Y$ is the projection. By definition of $\mathcal{M}_{S}(f)$, we have

$$
\partial \mathcal{M}_{S}(f) \cap S T B_{Y}=\overline{\left.\left\{\operatorname{grad}_{x} f\right] \mid x \in B_{Y} \backslash \operatorname{Crit}(f)\right\}}+\varepsilon \sum_{i, j} \hat{g}_{j i} \pi^{-1}\left(\overline{\mathcal{D}_{p_{i}} \cap \mathcal{A}_{q_{j}}}\right) .
$$

We recall that $\mathcal{A}_{p_{i}}^{-}=\mathcal{D}_{p_{i}}$ and $\mathcal{D}_{q_{j}}^{-}=\mathcal{A}_{q_{j}}$. The involution $T: Y^{2} \rightarrow Y^{2},(x, y) \rightarrow(y, x)$ induces maps $T^{\prime}:\left(\mathcal{D}_{p_{i}} \times \mathcal{A}_{q_{j}}\right) \cap\left((Y \backslash \infty)^{2} \backslash \Delta\right) \rightarrow\left(\mathcal{A}_{q_{j}} \times \mathcal{D}_{p_{i}}\right) \cap\left((Y \backslash \infty)^{2} \backslash \Delta\right)$ and $T_{C_{2}(Y)}: C_{2}(Y) \rightarrow C_{2}(Y)$. With our orientation conventions, $T^{\prime}$ is orientationpreserving so that $T_{C_{2}(Y)}$ is orientation-preserving from $\varepsilon \pi^{-1}\left(\overline{\mathcal{D}_{p_{i}} \cap \mathcal{A}_{q_{j}}}\right)$ to the boundary part $\pm \pi^{-1}\left(\overline{\mathcal{D}_{p_{i}} \cap \mathcal{A}_{q_{j}}}\right)$ of the closure of $\left(\mathcal{A}_{q_{j}} \times \mathcal{D}_{p_{i}}\right) \cap\left((Y \backslash \infty)^{2} \backslash \Delta\right)$, which therefore reads $-\varepsilon \pi^{-1}\left(\overline{\mathcal{D}_{p_{i}} \cap \mathcal{A}_{q_{j}}}\right)$ since $T_{C_{2}(Y)}$ reverses the orientation of the fiber of $S T B_{Y}$. Then we have

$$
\begin{aligned}
\partial \mathcal{M}_{S} & ( \pm f) \cap S T B_{Y} \\
& =\overline{\left.\left\{\operatorname{grad}_{x} f\right],\left[-\operatorname{grad}_{x} f\right]\right\}}+\varepsilon \sum_{i, j} \hat{g}_{j i} \pi^{-1}\left(\overline{\mathcal{D}_{p_{i}} \cap \mathcal{A}_{q_{j}}}\right)-\varepsilon \sum_{i, j} \hat{g}_{j i} \pi^{-1}\left(\overline{\mathcal{D}_{p_{i}} \cap \mathcal{A}_{q_{j}}}\right) \\
& =\overline{\left.\left\{\operatorname{grad}_{x} f\right],\left[-\operatorname{grad}_{x} f\right]\right\}} .
\end{aligned}
$$

Lemma 5.19 $\partial \mathcal{M}_{S}( \pm f)=c(\operatorname{grad} f)$ for any admissible Morse function $f$.

Proof Since $\left.\operatorname{grad} f\right|_{N(\infty ; Y)}=a$, if $(x, u) \in \partial \mathcal{M}_{S}( \pm f) \cap\left((Y \backslash \infty) \times S T_{\infty} Y\right)$, then $u \in\{a,-a\}$. On the other hand, $\partial \mathcal{M}_{S}( \pm f) \cap\left(\{x\} \times S T_{\infty} Y\right)=\{(x, a),(x,-a)\}$ as sets for any $x \notin \operatorname{Crit}(f)$. Since $\partial \mathcal{M}_{S}( \pm f)$ is a 3-cycle, we have

$$
\partial \mathcal{M}_{S}(f) \cap\left((Y \backslash \infty) \times S T_{\infty} Y\right)=-(Y \backslash \infty) \times\{a,-a\}
$$

with our orientation conventions. We have $\mathcal{M}_{S}( \pm f) \backslash S T B_{Y}=p_{Y}^{-1}(\{a,-a\})$ by a similar argument. Due to this fact and Lemma 5.18, the proof is complete.

We follow the notation $a_{1}, \ldots, a_{3 n}, f_{1}, \ldots, f_{3 n}$ as in Section 4.3. Let $C_{2 n}(Y)$ be the compactification of $\breve{C}_{2 n}(Y)$ given in [8, Section 3]. In the following proposition, the notion "generic $f_{1}, \ldots, f_{3 n}$ " means that $\partial C_{2 n}(Y) \cap\left(\bigcap_{i} P_{i}(\Gamma)^{-1} \mathcal{M}_{S}\left( \pm f_{i}\right)\right)=\varnothing$ for any $\Gamma \in \mathcal{E}_{n}$. 
Remark 5.20 We can show that $\partial C_{2 n}(Y) \cap\left(\bigcap_{i} P_{i}(\Gamma)^{-1} \mathcal{M}_{S}\left( \pm f_{i}\right)\right)=\varnothing$ for generic $f_{1}, \ldots, f_{3 n}$ by an argument similar to Lemma 4.2 in [14].

Proof of Theorem 4.7 We have that $\operatorname{grad} f_{1}, \ldots, \operatorname{grad} f_{3 n}$ are admissible vector fields. We take a propagator $\omega_{C}\left(\operatorname{grad} f_{i}\right)$ whose support is concentrated near $\mathcal{M}_{S}\left( \pm f_{i}\right)$ for each $i \in \underline{3 n}$. Since $f_{1}, \ldots, f_{3 n}$ are generic, it is possible to choose these propagators $\omega_{C}\left(\operatorname{grad} f_{1}\right), \ldots, \omega_{C}\left(\operatorname{grad} f_{3 n}\right)$ satisfying $\bigwedge_{i} P_{i}(\Gamma)^{*} \omega\left(\operatorname{grad} f_{i}\right) \equiv 0$ near the boundary of $C_{2 n}(Y)$ for any $\Gamma \in \mathcal{E}_{n}$. Then

$$
\#\left(\bigcap_{i} P_{i}(\Gamma)^{-1} \mathcal{M}\left( \pm f_{i}\right)\right)=\#\left(\bigcap_{i} P_{i}(\Gamma)^{-1} \mathcal{M}_{S}\left( \pm f_{i}\right)\right)
$$

for any $\Gamma \in \mathcal{E}_{n}$. By intersection theory, we have

$$
\int_{C_{2 n}(Y)} \bigwedge_{i} P_{i}(\Gamma)^{*} \omega_{C}\left(\operatorname{grad} f_{i}\right)=\frac{1}{2^{3 n}} \#\left(\bigcap_{i} P_{i}(\Gamma)^{-1} \mathcal{M}_{S}\left( \pm f_{i}\right)\right)
$$

for any $\Gamma \in \mathcal{E}_{n}$. Therefore,

$$
\begin{aligned}
z_{n}^{\mathrm{KKT}}(Y) & =z_{n}\left(\left(\omega\left(\operatorname{grad} f_{i}\right)\right)_{i \in \underline{3 n}}\right)-\widetilde{z}_{n}^{\text {anomaly }}\left(\left(\omega\left(\operatorname{grad} f_{i}\right)\right)_{i \in \underline{3 n}}\right) \\
& =\sum_{\Gamma \in \mathcal{E}_{n}}\left(\int_{C_{2 n}(Y)} \bigwedge_{i} P_{i}(\Gamma)^{*} \omega_{C}\left(\operatorname{grad} f_{i}\right)\right)[\Gamma]-\widetilde{z}_{n}^{\text {anomaly }}\left(\left(\omega\left(\operatorname{grad} f_{i}\right)\right)_{i \in \underline{3 n}}\right) \\
& =\sum_{\Gamma \in \mathcal{E}_{n}} \frac{1}{2^{3 n}} \#\left(\bigcap_{i} P_{i}(\Gamma)^{-1} \mathcal{M}_{S}\left( \pm f_{i}\right)\right)[\Gamma]-\widetilde{z}_{n}^{\text {anomaly }}\left(\left(\omega\left(\operatorname{grad} f_{i}\right)\right)_{i \in \underline{3 n}}\right) \\
& =\sum_{\Gamma \in \mathcal{E}_{n}} \frac{1}{2^{3 n}} \#\left(\bigcap_{i} P_{i}(\Gamma)^{-1} \mathcal{M}\left( \pm f_{i}\right)\right)[\Gamma]-\widetilde{z}_{n}^{\text {anomaly }}\left(\left(\omega\left(\operatorname{grad} f_{i}\right)\right)_{i \in \underline{3 n}}\right) .
\end{aligned}
$$

\section{Appendix: Another proof of Proposition 5.3 when $n=1$}

In this section, we give a more direct proof of Proposition 5.3 when $n=1$. Note that $\mathcal{A}_{1}(\varnothing)=\mathbb{Q}[\theta]$ and $\# \mathcal{E}_{1}=8$. We first prepare some notation. Let $\pi_{1}: F_{X^{\prime}} \rightarrow S T^{v} X^{\prime}$ be the tangent bundle along the fiber of $\pi_{2}: S T^{v} X^{\prime} \rightarrow X^{\prime}$.

Let $e\left(F_{X^{\prime}}\right) \in H^{2}\left(S T^{v} X^{\prime}\right)$ be the Euler class of $F_{X^{\prime}}$, and let $p_{1}\left(F_{X^{\prime}}\right) \in H^{4}\left(S T^{v} X^{\prime}\right)$ be the first Pontrjagin class of $F_{X^{\prime}}$. By a standard argument, the Chern-Weil theory 
for example, we have $p_{1}\left(F_{X^{\prime}}\right)=e\left(F_{X^{\prime}}\right)^{2}$; eg see Corollary 15.8 of [13]. Then

$$
\begin{aligned}
X_{1}\left(T^{v} X^{\prime} ;\left(W_{i}^{\prime}\right)_{i \in \underline{3 n}}\right) & =8\left(\int_{S T^{v} X^{\prime}} W_{1}^{\prime} \wedge W_{2}^{\prime} \wedge W_{3}^{\prime}\right)[\theta] \\
& =\int_{S T^{v} X^{\prime}} e\left(F_{X^{\prime}}\right)^{3}[\theta] \\
& =\int_{S T^{v} X^{\prime}} e\left(F_{X^{\prime}}\right) p_{1}\left(F_{X^{\prime}}\right)[\theta] \\
& \stackrel{(*)}{=} \int_{S T^{v} X^{\prime}} e\left(F_{X^{\prime}}\right) \pi_{2}^{*} p_{1}\left(T X^{\prime}\right)[\theta] \\
& =2 \int_{X^{\prime}} p_{1}\left(T X^{\prime}\right)[\theta] \\
& =6[\theta] \operatorname{Sign} X^{\prime} .
\end{aligned}
$$

Equation (*) is given by $\underline{\mathbb{R}} \oplus F_{X^{\prime}}=\pi^{*} T^{v} X^{\prime}$ and $\mathbb{R} \oplus T^{v} X^{\prime}=T X^{\prime}$. Then we have $X_{1}\left(T^{v} X^{\prime} ;\left(W_{i}^{\prime}\right)_{i \in \underline{3 n}}\right)=6[\theta] \operatorname{Sign} X^{\prime}$.

\section{References}

[1] M Atiyah, On framings of 3-manifolds, Topology 29 (1990) 1-7 MR

[2] S Axelrod, I M Singer, Chern-Simons perturbation theory, from "Proceedings of the XXth International Conference on Differential Geometric Methods in Theoretical Physics, Vol 1, 2" (S Catto, A Rocha, editors), World Sci. Publ., River Edge, NJ (1992) 3-45 MR

[3] K Fukaya, Morse homotopy and Chern-Simons perturbation theory, Comm. Math. Phys. 181 (1996) 37-90 MR

[4] M Futaki, On Kontsevich's configuration space integral and invariants of 3-manifolds, master's thesis, Univ. of Tokyo (2006)

[5] R Kirby, P Melvin, Canonical framings for 3-manifolds, from "Proceedings of 6th Gökova Geometry-Topology Conference" (S Akbulut, T Ander, R J Stern, editors), Turkish J. Math. 23 (1999) 89-115 MR

[6] M Kontsevich, Feynman diagrams and low-dimensional topology, from "First European Congress of Mathematics, Vol II" (A Joseph, F Mignot, F Murat, B Prum, R Rentschler, editors), Progr. Math. 120, Birkhäuser, Basel (1994) 97-121 MR

[7] G Kuperberg, D P Thurston, Perturbative 3-manifold invariants by cut-and-paste topology, preprint (1999) arXiv

[8] C Lescop, On the Kontsevich-Kuperberg-Thurston construction of a configurationspace invariant for rational homology 3-spheres, preprint (2004) arXiv 
[9] C Lescop, Splitting formulae for the Kontsevich-Kuperberg-Thurston invariant of rational homology 3-spheres, preprint (2004) arXiv

[10] C Lescop, Surgery formulae for finite type invariants of rational homology 3-spheres, Algebr. Geom. Topol. 9 (2009) 979-1047 MR

[11] C Lescop, A formula for the $\Theta$-invariant from Heegaard diagrams, Geom. Topol. 19 (2015) 1205-1248 MR

[12] C Lescop, On homotopy invariants of combings of three-manifolds, Canad. J. Math. 67 (2015) 152-183 MR

[13] J W Milnor, J D Stasheff, Characteristic classes, Annals of Mathematics Studies 76, Princeton University Press (1974) MR

[14] T Watanabe, Higher order generalization of Fukaya's Morse homotopy invariant of 3-manifolds, I: Invariants of homology 3-spheres, preprint (2012) arXiv

[15] J H C Whitehead, On the groups $\pi_{r}\left(V_{n, m}\right)$ and sphere-bundles, Proc. London Math. Soc. 48 (1944) 243-291 MR Correction in 49 (1947) 479-481

Research Institute for Mathematical Sciences, Kyoto University, The Mathematical Society of Japan,

Kitashirakawa-Oiwake cho, Sakyo-ku, Kyoto city 606-8502, Japan

shimizu@kurims.kyoto-u.ac.jp

Received: 5 November $2013 \quad$ Revised: 19 March 2016 
